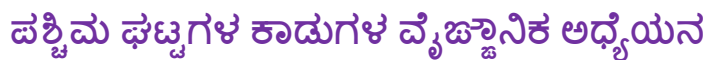

T.M. Geethanjali a,

\title{
A Scientific study of Forests of the Western Ghats
}

a Department of Kannada, Karnataka State Open University, Mukhtagangotri, Mysuru-560007, Karnataka, India

*Corresponding author Email: tm.geethanjali@gmail.com

DOI: https://doi.org/10.34256/ijmrd2137

Received: 09-07-2021; Revised: 15-08-2021; Accepted: 17-08-2021; Published: 18-08-2021

Abstract: The Western Ghats in India's Deccan Plateau has been recognized as one of the eight "hottest hotspots" of biological diversity in the world. UNESCO has identified this region as a World Heritage Site. In Maharashtra, this mountain range is known as 'Sahyadri', 'Dang forests' in Gujarat, 'Nilgiris' in Tamil Nadu and Kerala, and 'Malnad' in Karnataka. It stretches across the six Indian states of Gujrat, Maharashtra, Goa, Tamil Nadu, Kerala, Karnataka and two union territories including Daman and Diu and Pondicherry. These hills begin near the border of Gujarat and Maharashtra, south of the Tapti river, and end at Kanyakumari, at the southern tip of India, covering an incredible $1,60,000 \mathrm{sq} . \mathrm{km}$. In the $18^{\text {th }}$ and $19^{\text {th }}$ centuries, most of the people who classified the flora of these regions are hobbyists. Very few British Officers who conduct survey of this landscape release their books. A complete scientific study of this biodiversity hot-spot is yet to be undertaken. Until now, only a handful of scientists have undertaken a study of the Western Ghats. So, this present article expains a brief scientific study of the forests of the Western Ghats.

Keywords: Western Ghats, UNESCO, Forest, Deccan Plateau

Subject Specialization: Kannada

\section{తేరిభయయ}

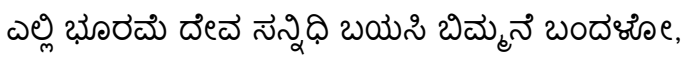

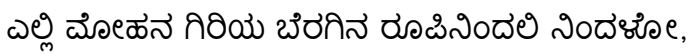

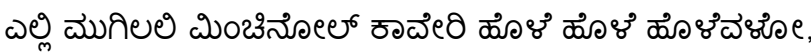

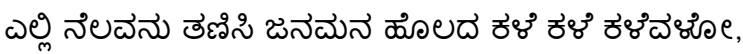

అల్లి ఆ కృడా నేండడాలా!

అల్లి శోడేదరర నాడెలా!

అల్లి శోడేదెర బిలడెలా!

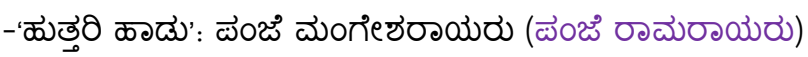

ஸेర్జితు

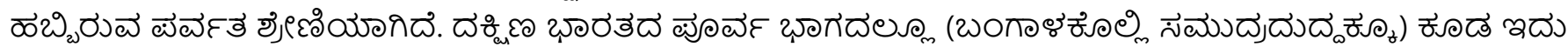
ळబిబ్బది. అల్లి ఇదను

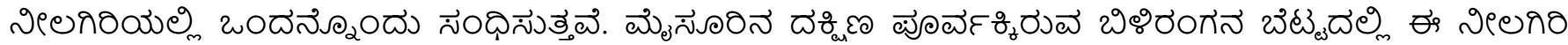

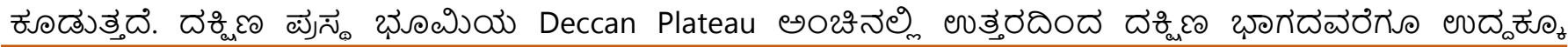




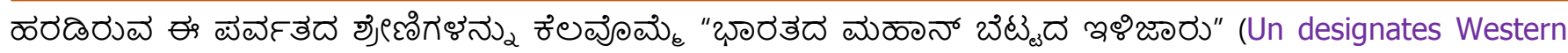

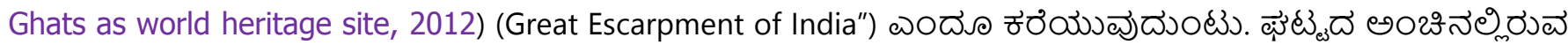

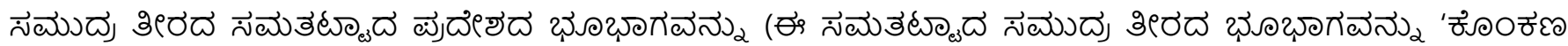
యూగగణ

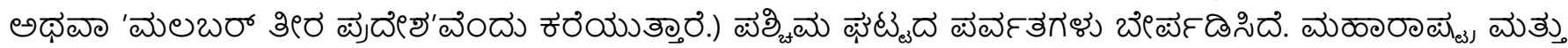

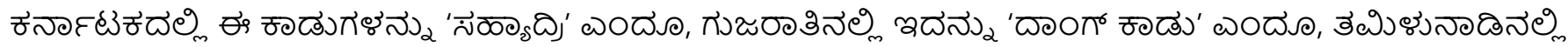
'నిలగిర యుల్ట'

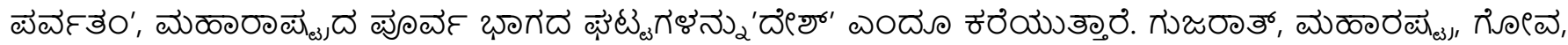

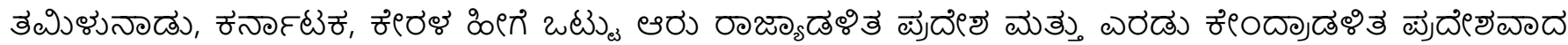

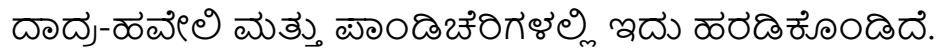

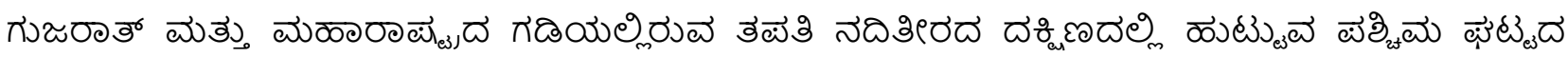

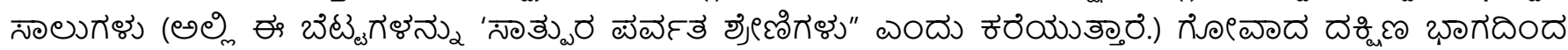

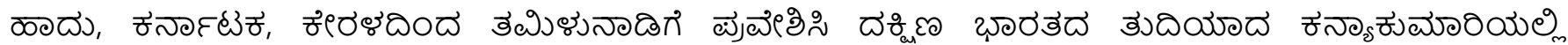

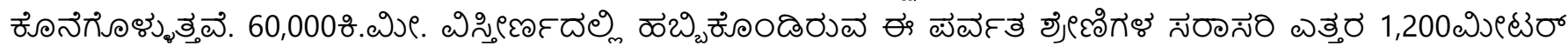

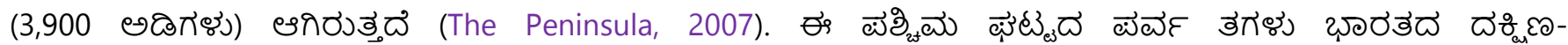

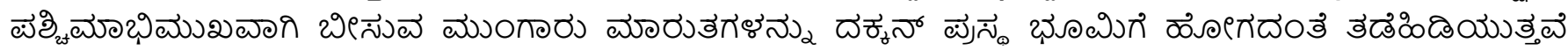

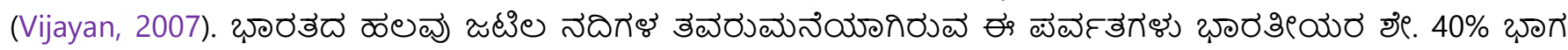

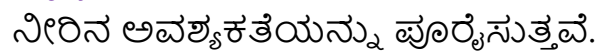

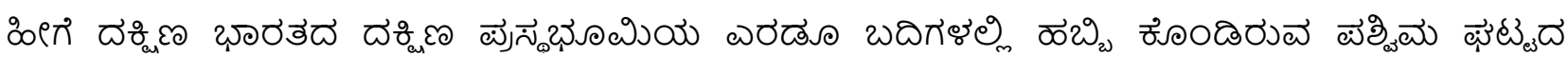

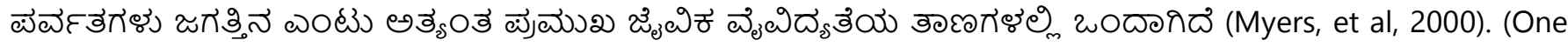

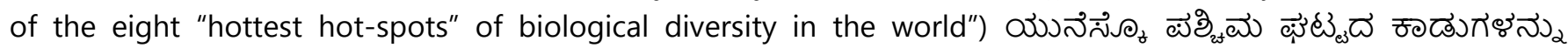

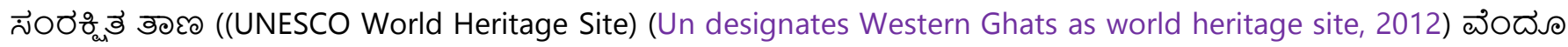

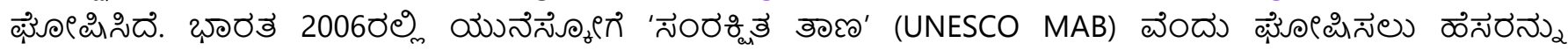

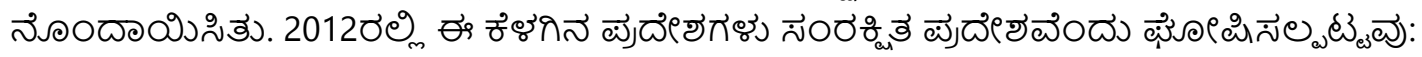

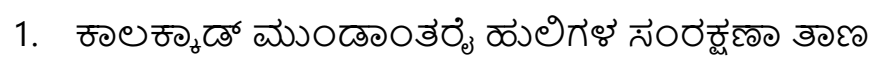

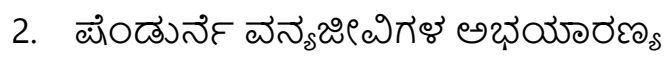

3. నియోఠ్యో

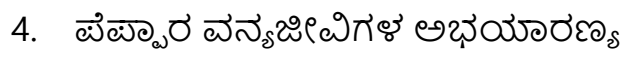

5. ెరరయయరో ळులిగిళ

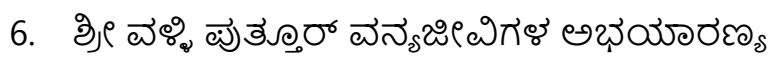

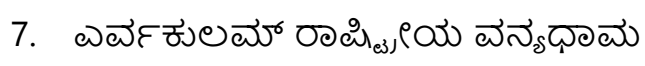

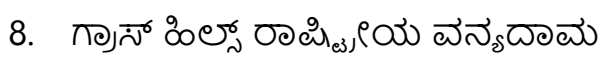

9. రరయనా छృల రృషిట్టి

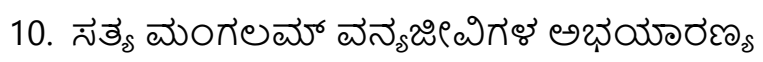

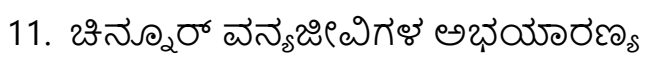

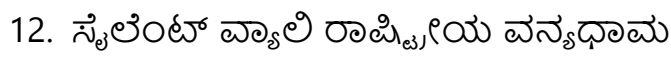

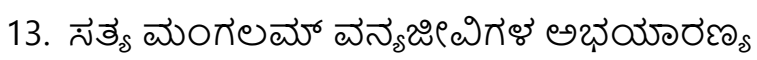

14.

15.

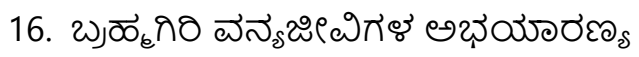

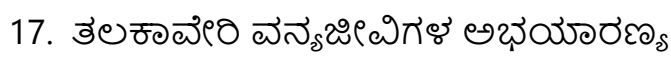

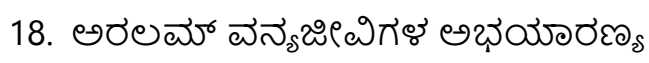




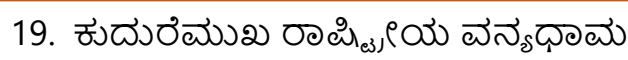

20.

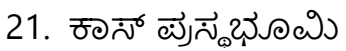

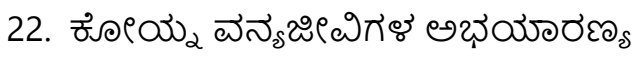

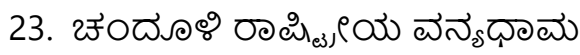

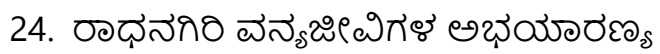

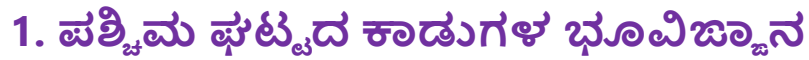

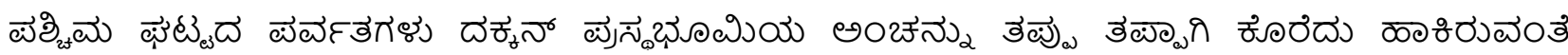

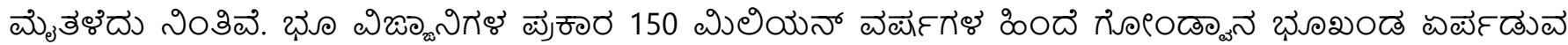

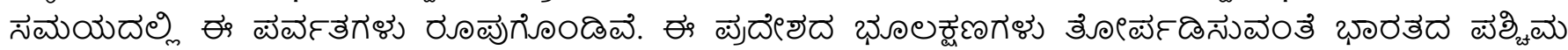

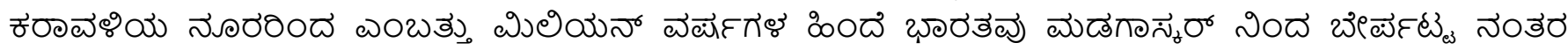

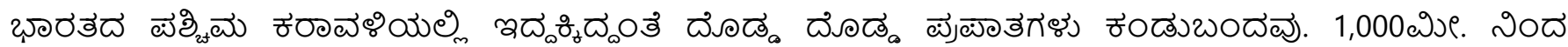

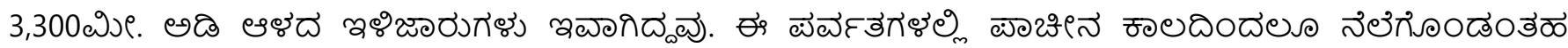

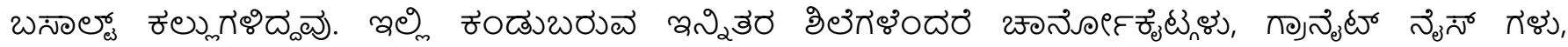

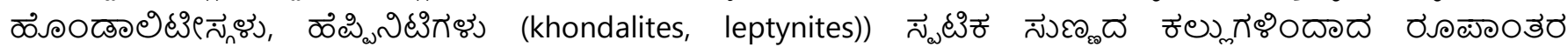

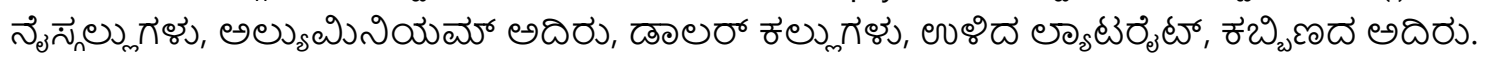

\section{2.}

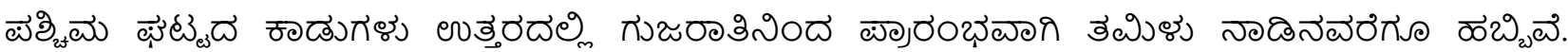

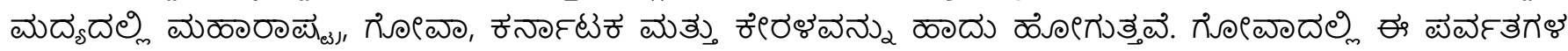

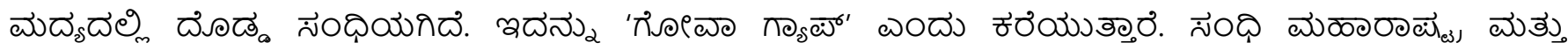

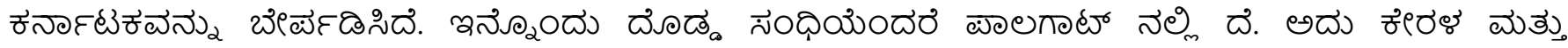

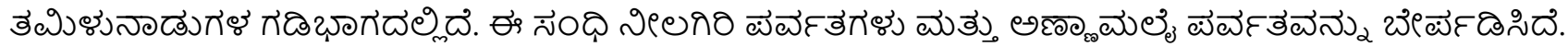

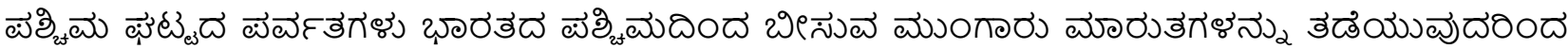

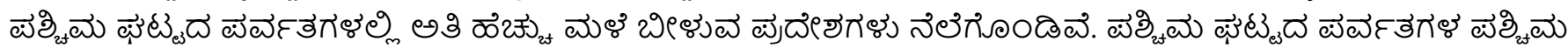

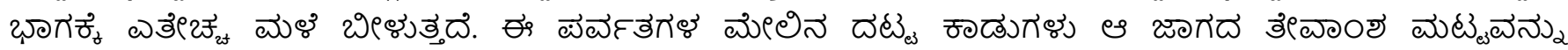

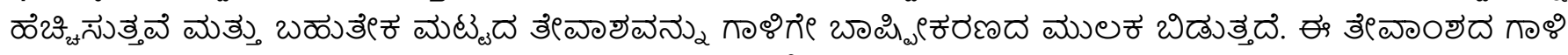

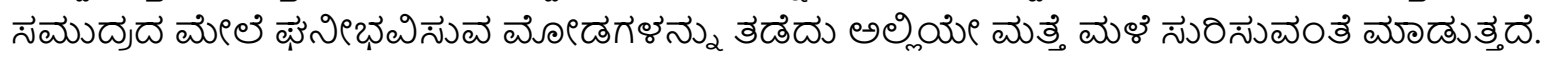

ఈన్నదిగరిగ

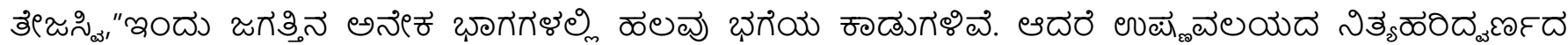

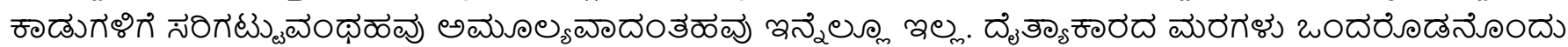

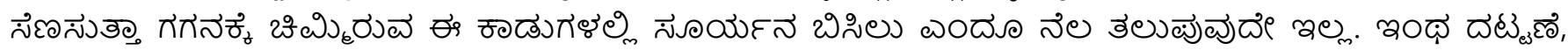

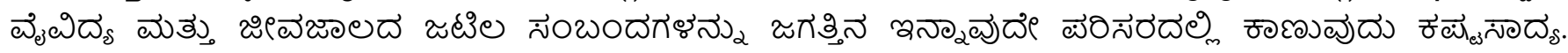

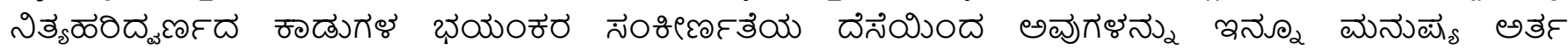

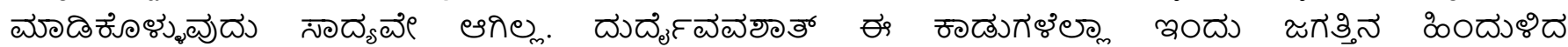

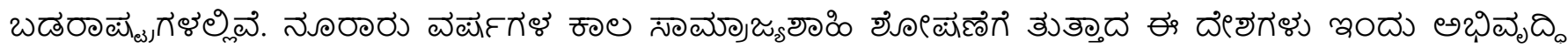

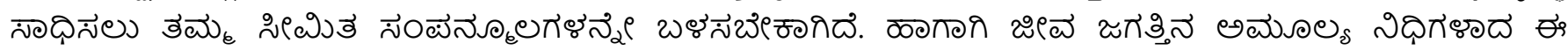

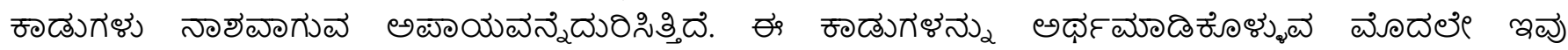

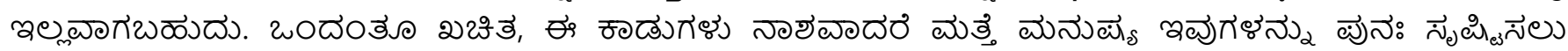

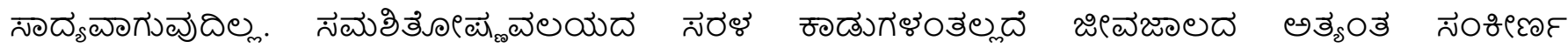

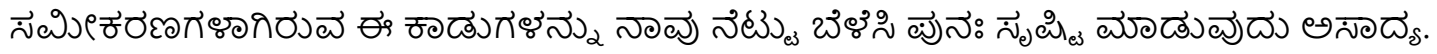

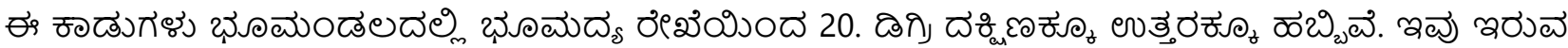

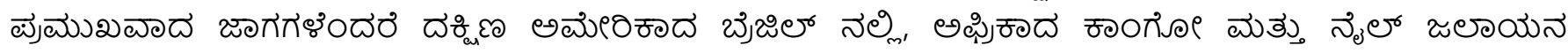

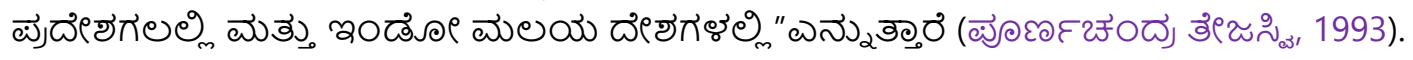




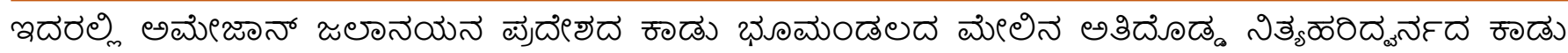

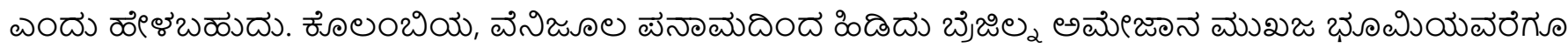

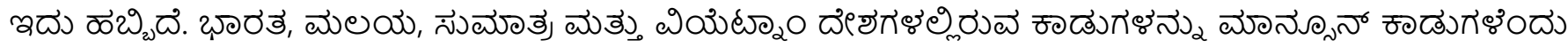
ఈరియు उల్తరి.

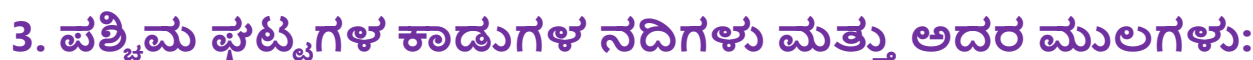

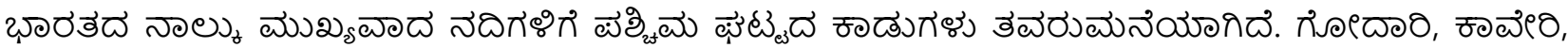
ఈృ $\vec{\omega}_{ణ \prime}$ శెఱిర

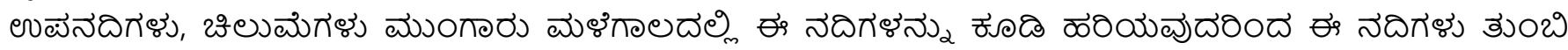

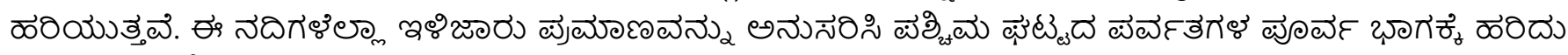

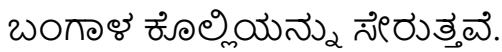

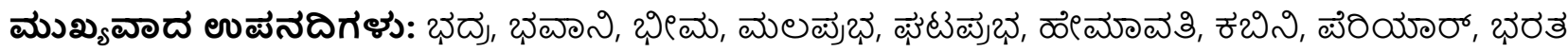

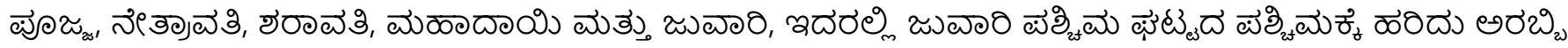

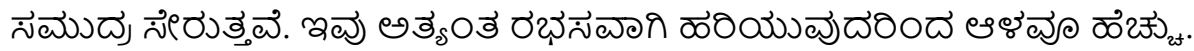

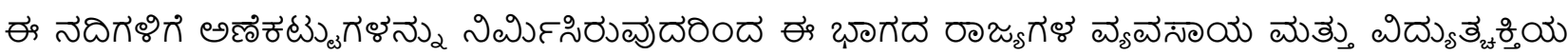

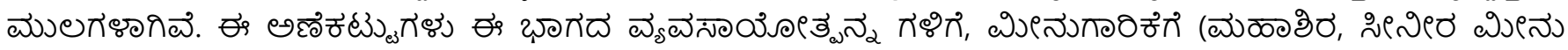

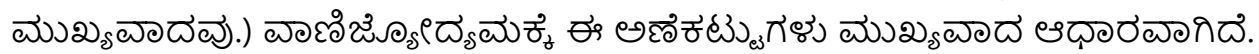

\section{యుدఖ్యळాదే అణిశటు}

శீలఱ్న్ -

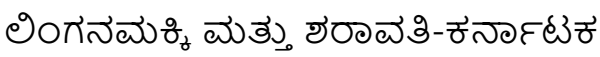

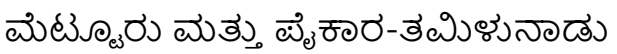

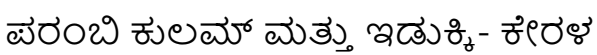

\section{మొगఖ్యळాదా జలळాతేగిళ}

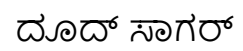

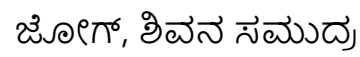

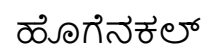

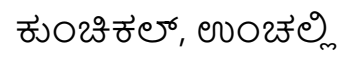

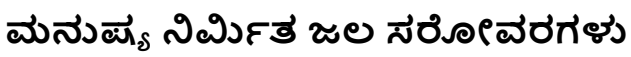

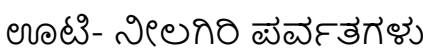

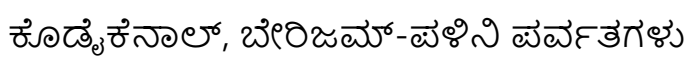

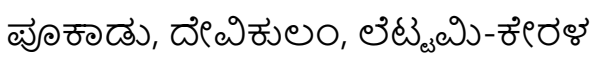

\section{4.}

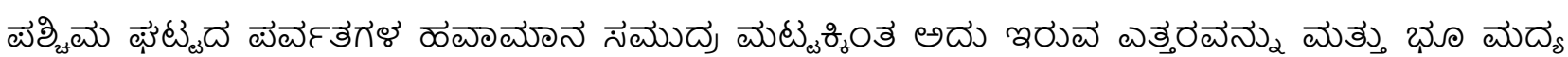

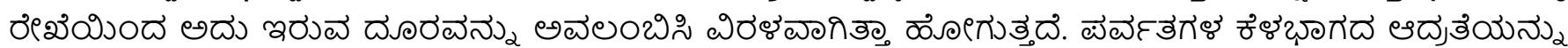

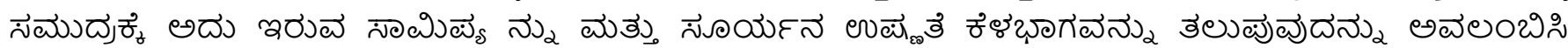

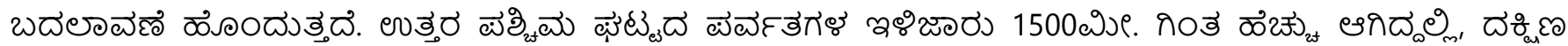

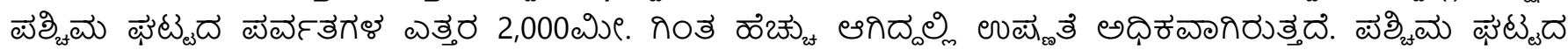

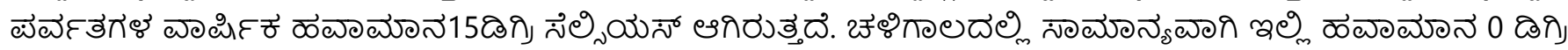

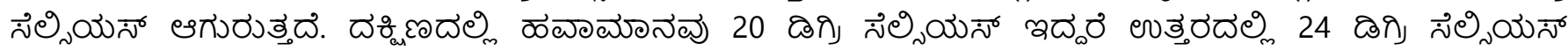

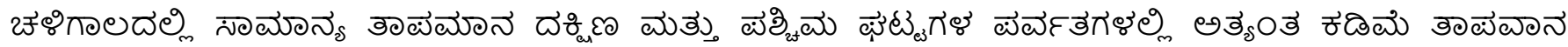

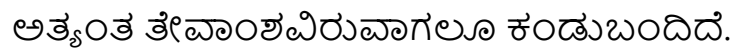

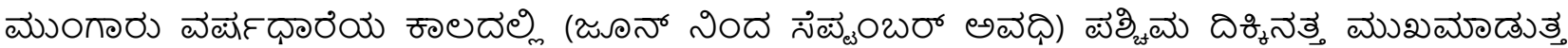

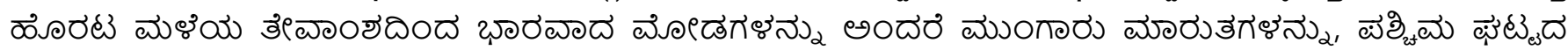

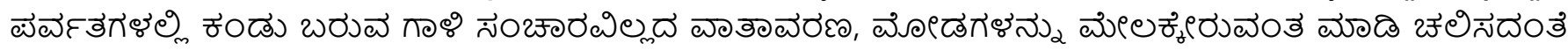

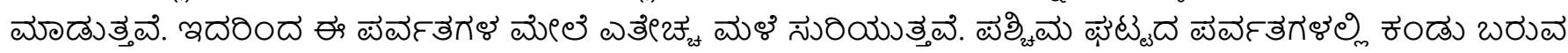




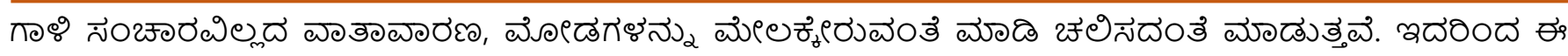

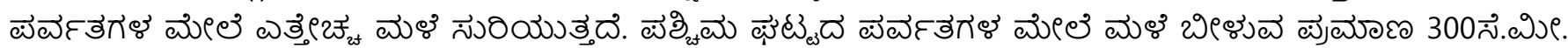

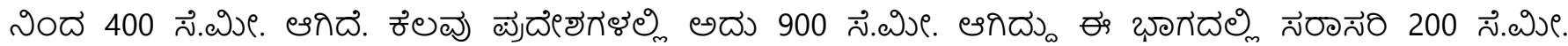

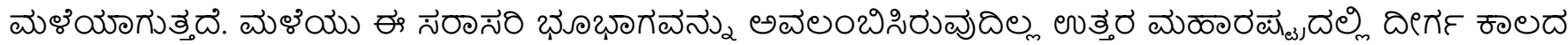

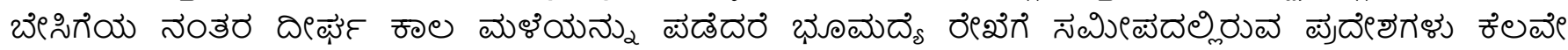
3ింగేళరల ఈడియి ముళ3యన్ను

\section{5.}

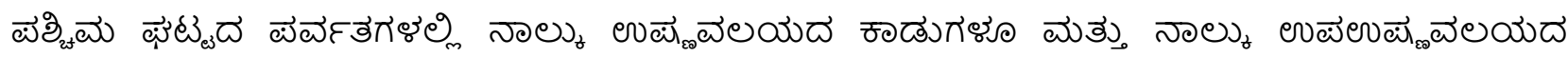

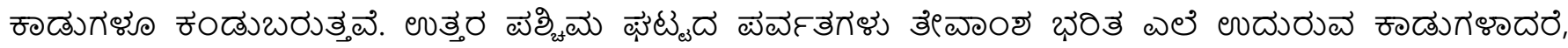

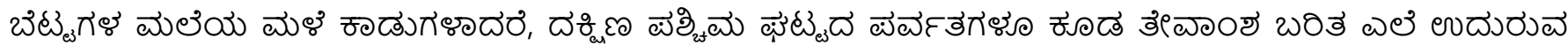

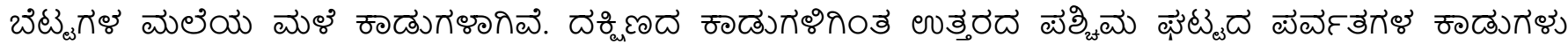

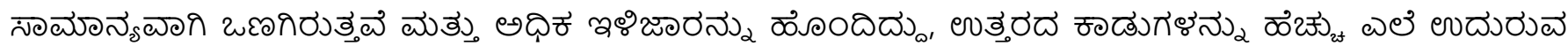

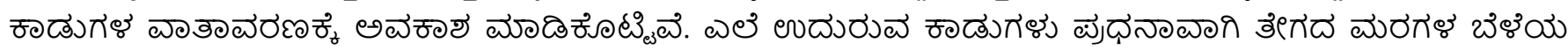

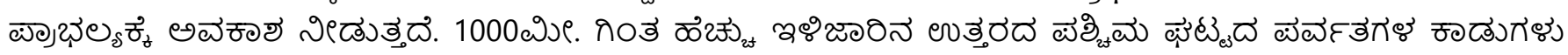

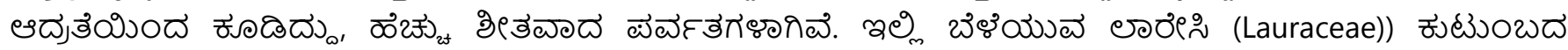

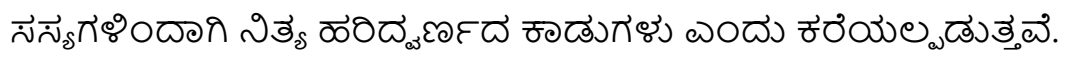

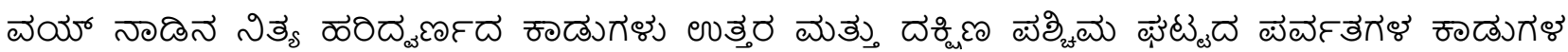

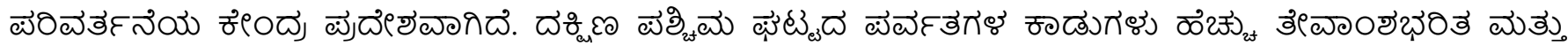
ळౌҒ్

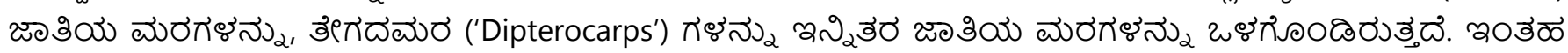

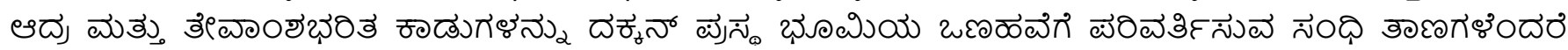
సేదా

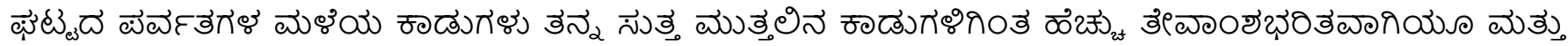

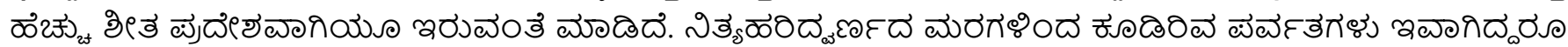

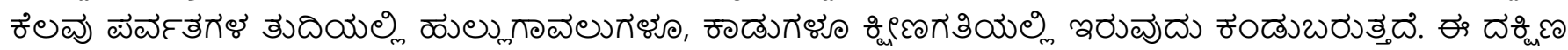

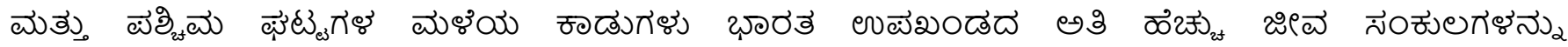

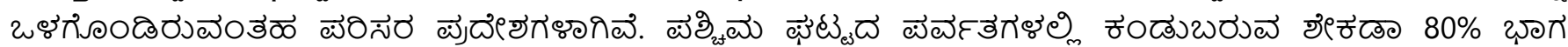

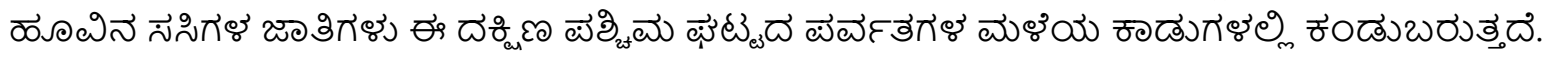

ఈనాళటళద

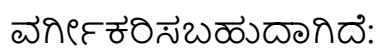

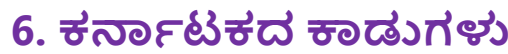

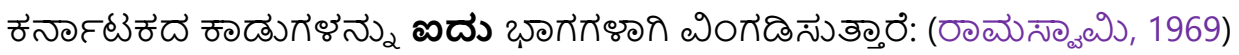

1.

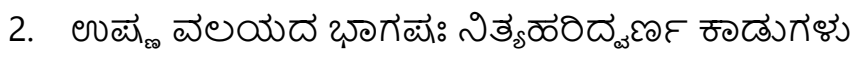

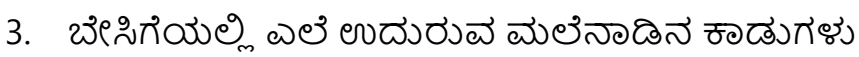

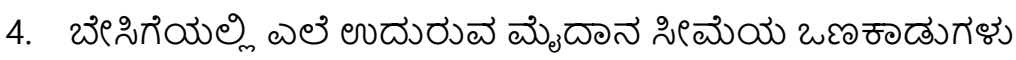

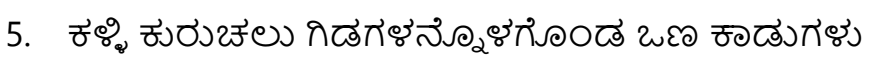

ఇదరల్లి 'లుడ్టణ

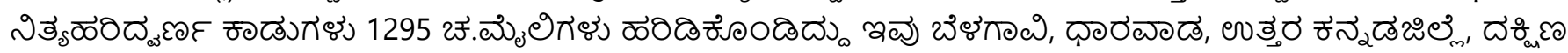

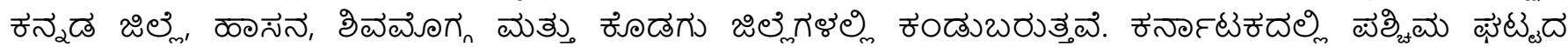

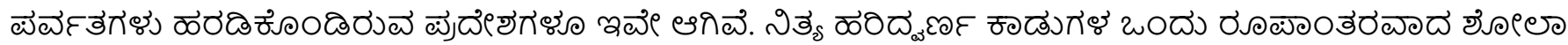

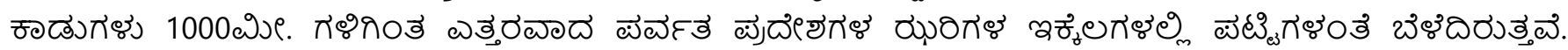

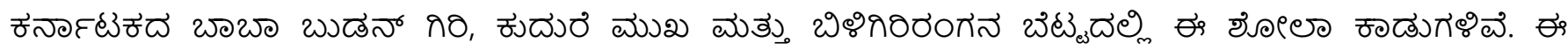




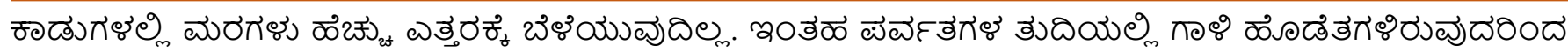

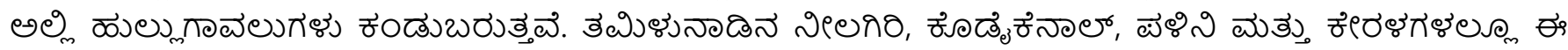

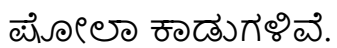

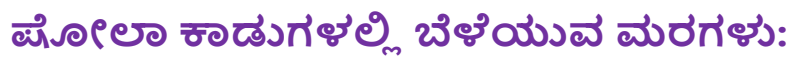

దిఠడ

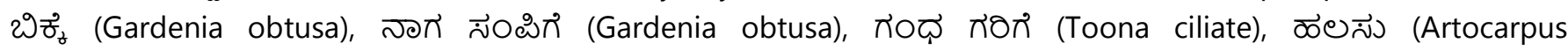

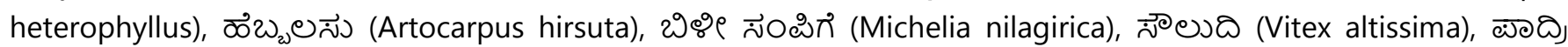
(Stereospermum chelonioides), (Canarium strictum), శణ

సెణ ఈృళతుడు యుర (Mallotus phillippenses).

ేనాళటేద

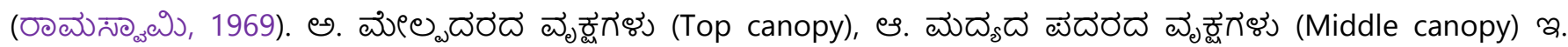

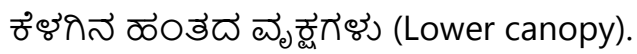

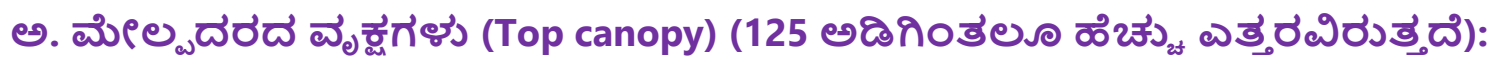

నอగกர்

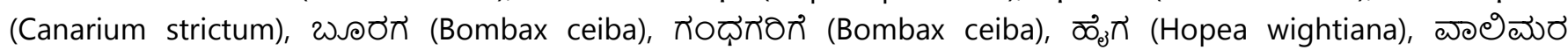

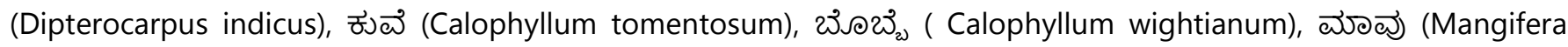

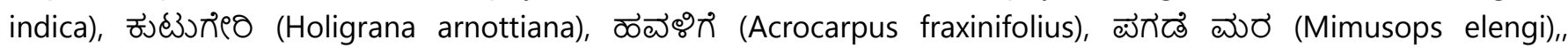

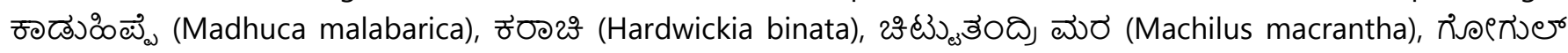

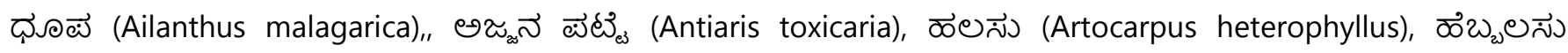

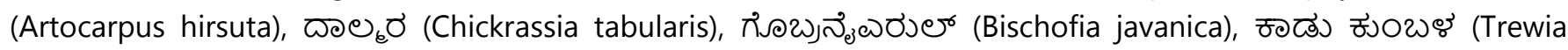

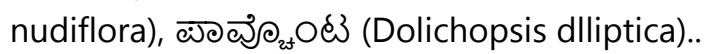

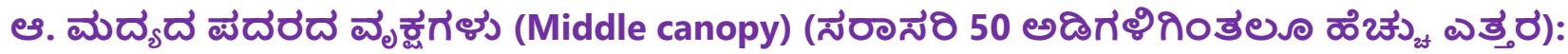

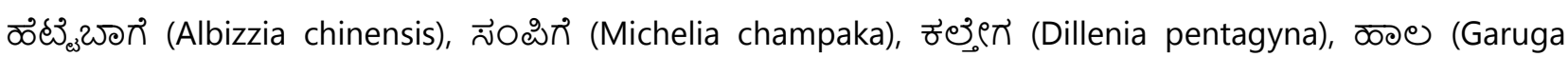

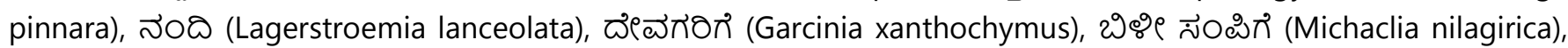

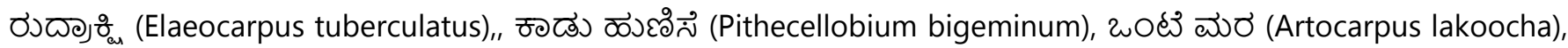

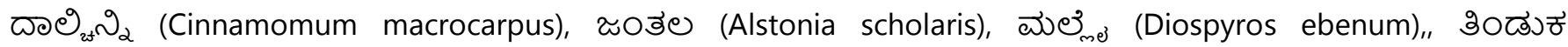

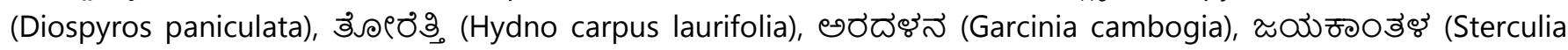

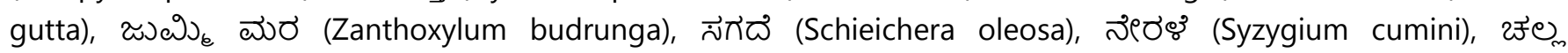
(Lagerstroemia speciosa), నెంజినే శోరెడు (Strychnos nux-vomica), ఉొదృయుర (Sterospermum chelonioides), గిలడృ

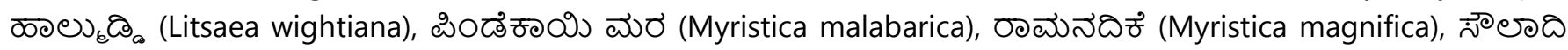
(Vitex altissima), $\vec{\sim} \jmath ర \varpi 0 న న_{2}$ (Guttiferae).

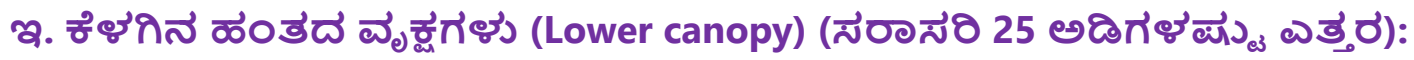

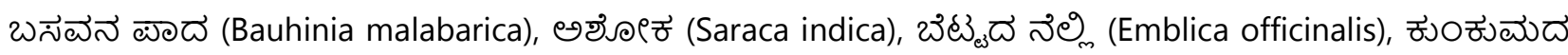

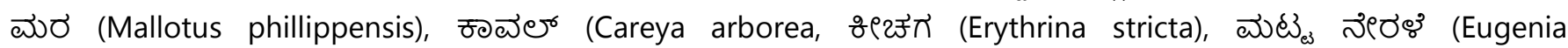

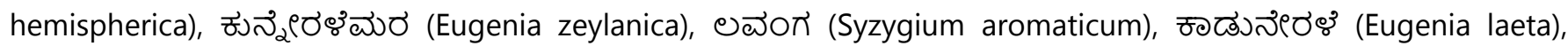

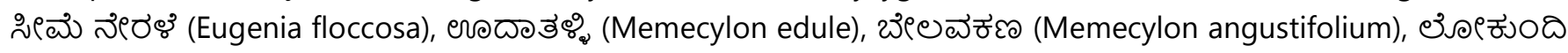
(Memecylon malabaricum), శరిబెలయు (Murraya koenigii, బిండుతెర (Trema orientalis), శృండి (Lonicera rainiflora),

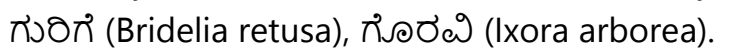




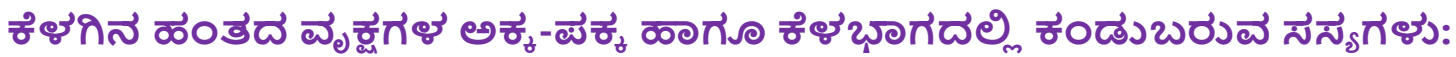

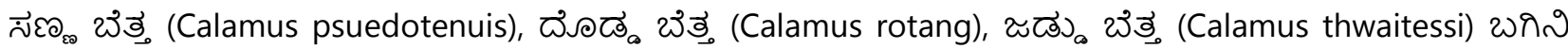

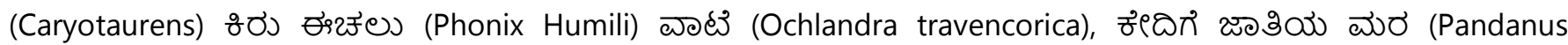
fureatusw and Pandanus canaranus), నిరతెంజి (Salix tetrasperma).

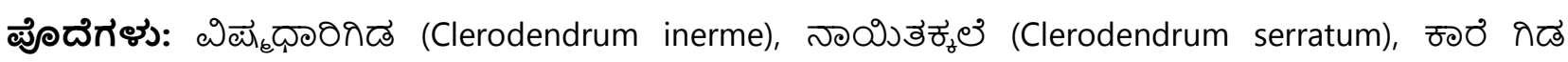

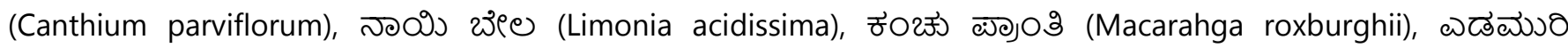

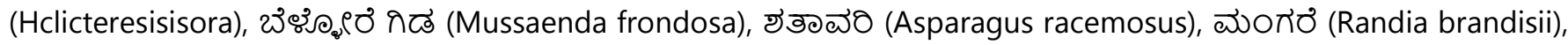
బిऽలదినె (Ardisia solancea).

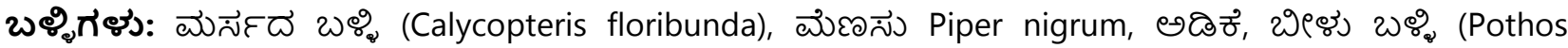

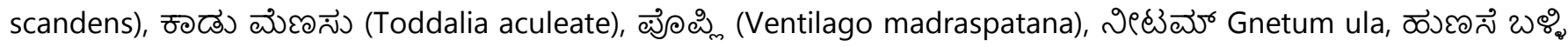
(Elaegnus latifolia), ळజ్జిలల (Elaeagnus kologa), శలి జదరి ळంబు (Clematis gouriana).

\section{7.}

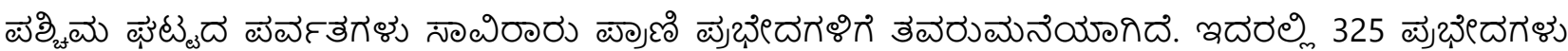

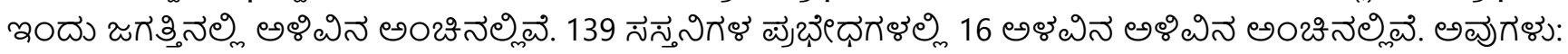

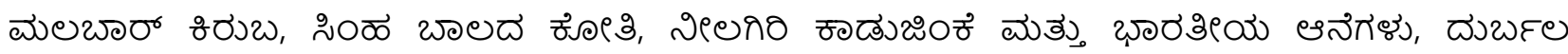

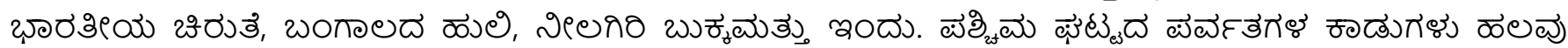

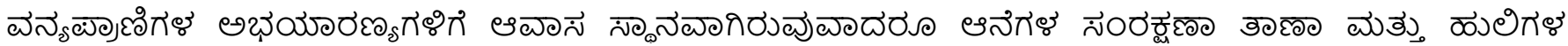

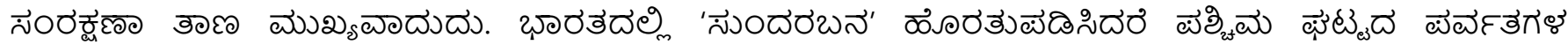

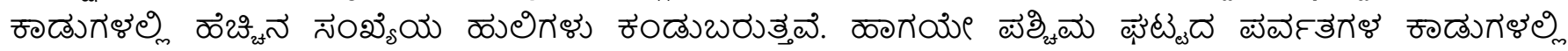

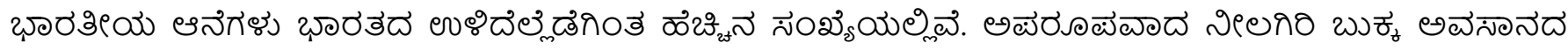

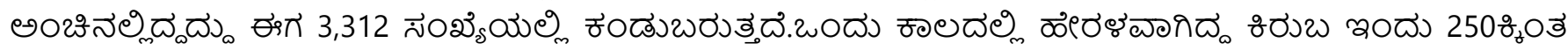

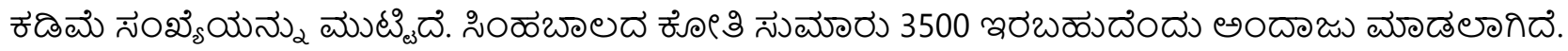

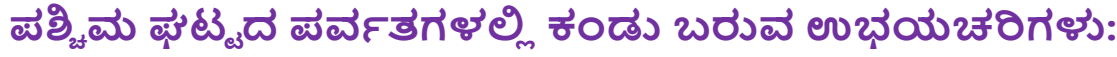

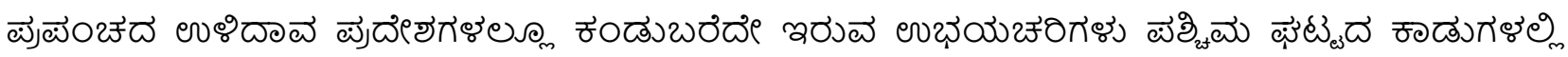

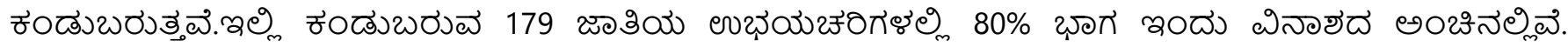

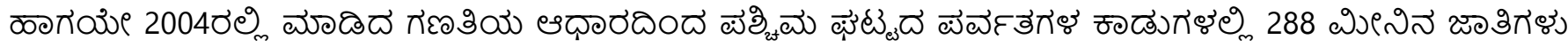

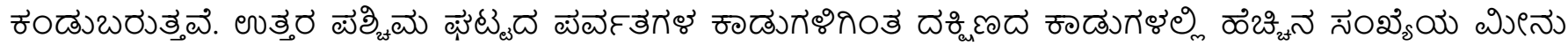

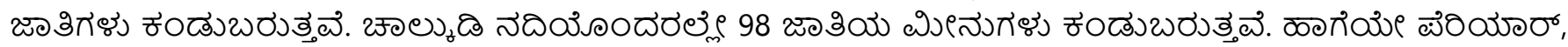

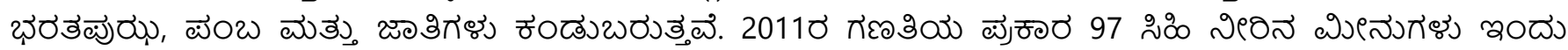
అ๗ాయద అంభినత్రియి.

ఉబభ్కియు

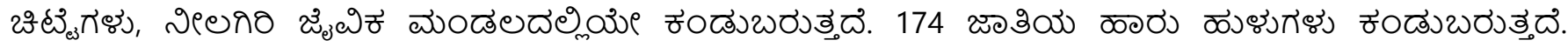

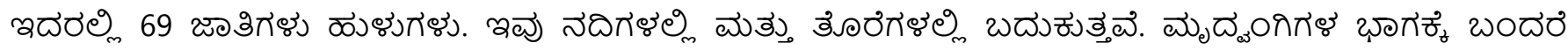

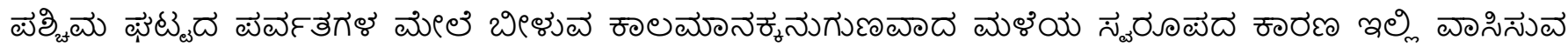

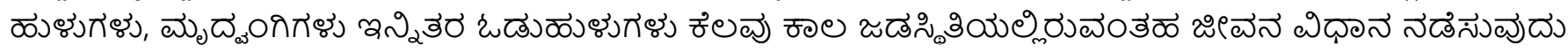
అనిథాయణ అాగిత్తు.

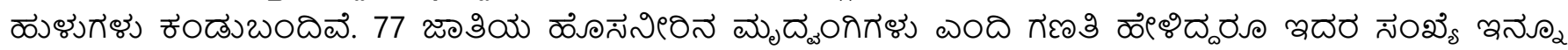
ळబఠ॰

\section{8.}

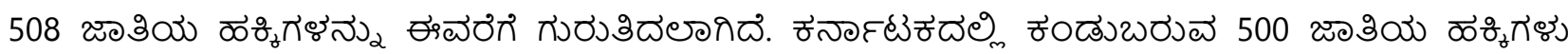
థౌ్రి

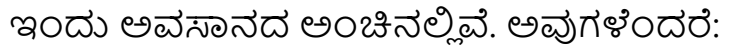




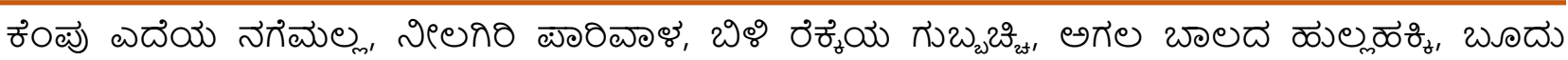

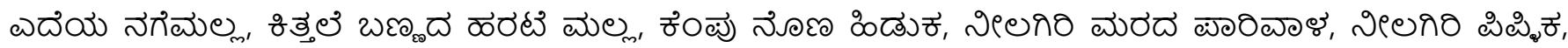

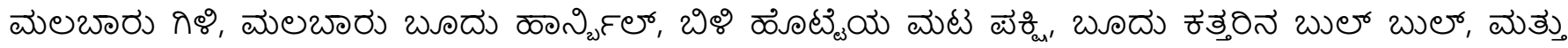

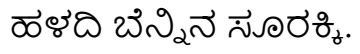

ఇదరలల్లి

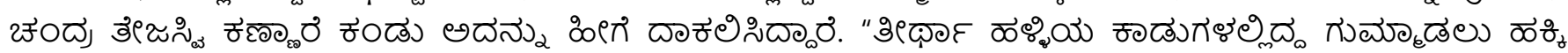

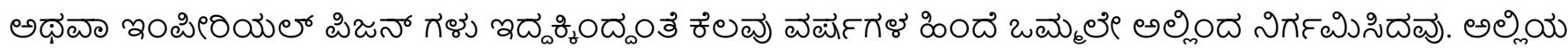

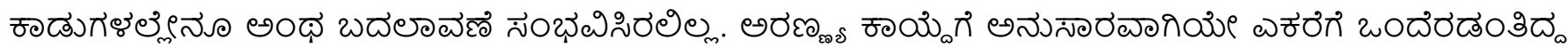

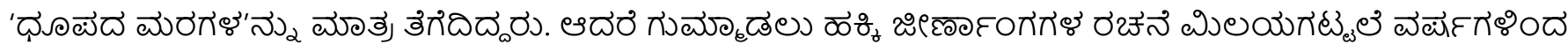

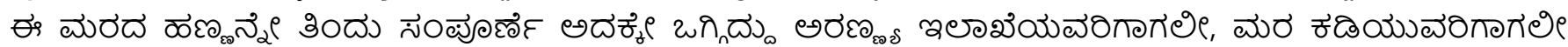

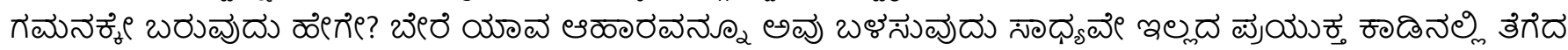

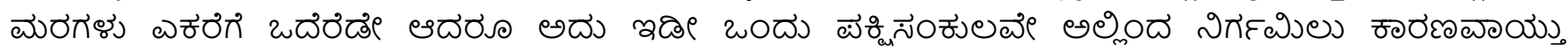
(

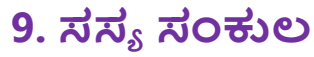

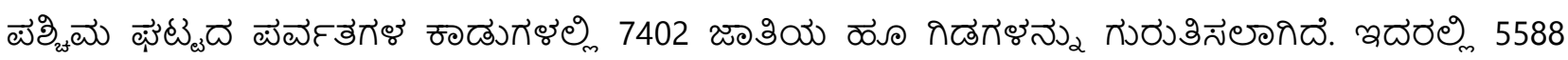
జలిగేృ

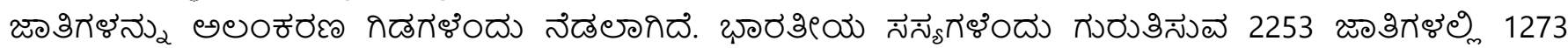
జల 3ిగెళ

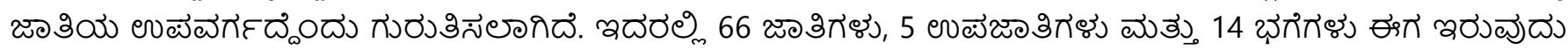

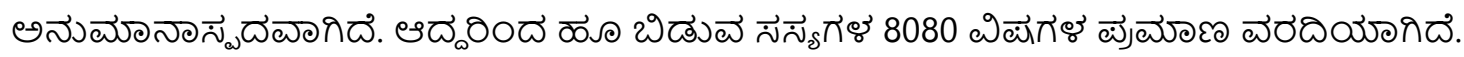

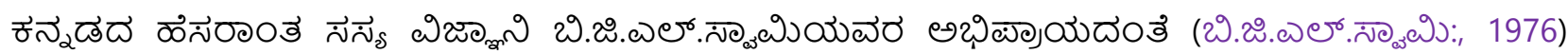
ేన్నడ నాడిన

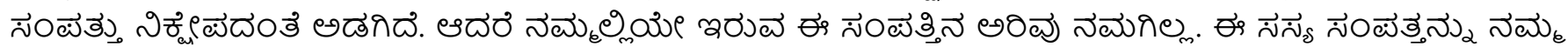

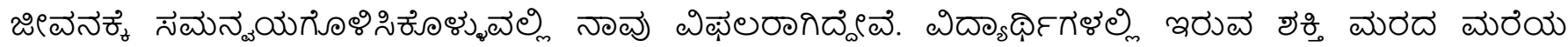

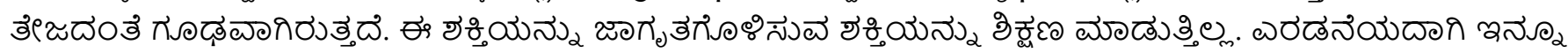

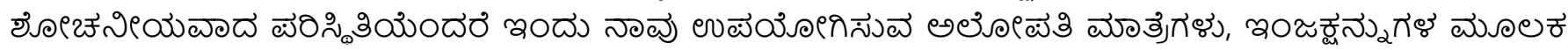

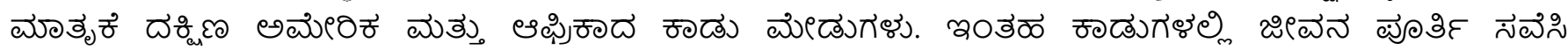

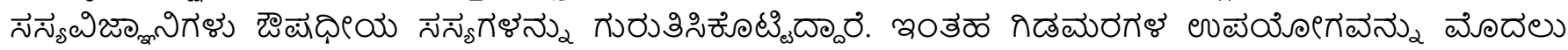

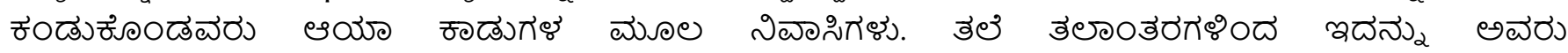

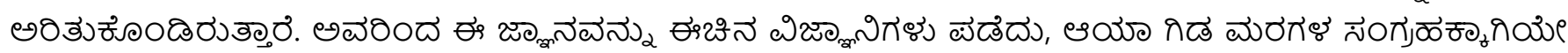

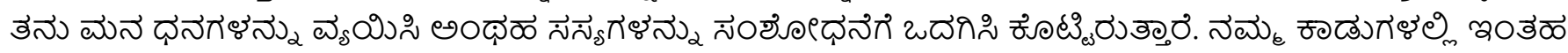

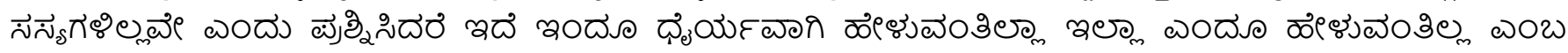

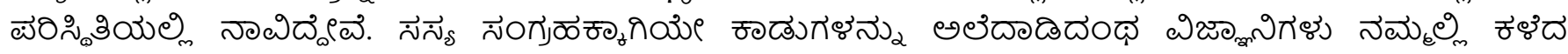

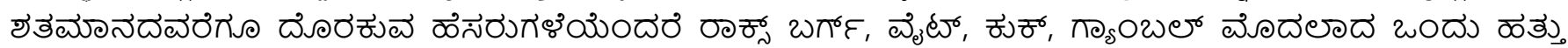

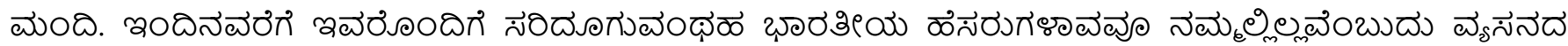
$\vec{\sim} \circ \pi 3$.

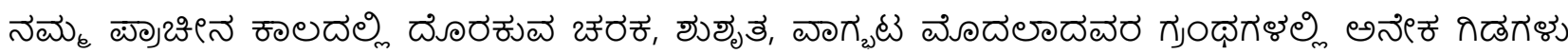

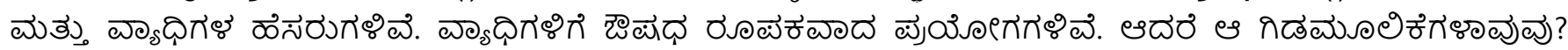

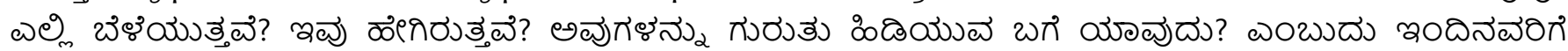

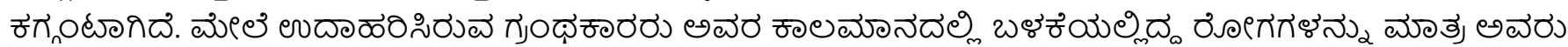

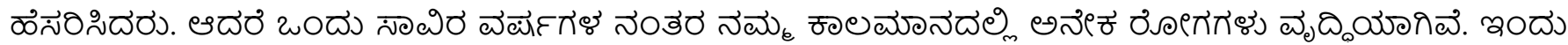

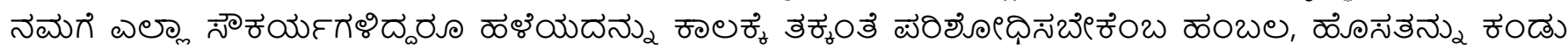

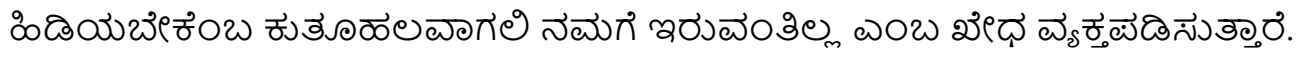

బి.జి.ఎలో సెట్టా

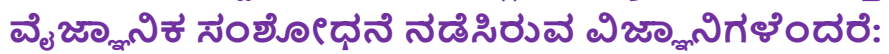

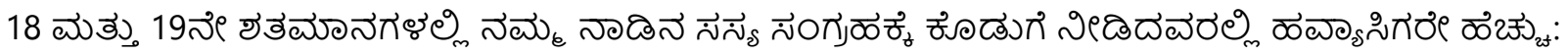
Indian J Multiling Res Dev., 2(3) (2021), 52-64| 59 
అ. గృ్యంబలో

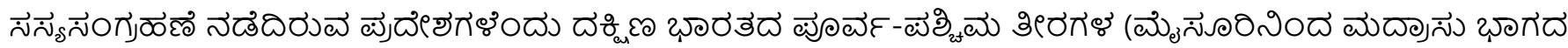

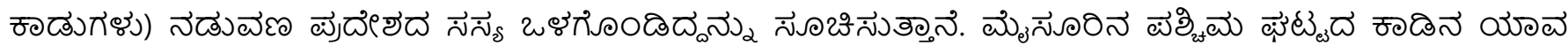

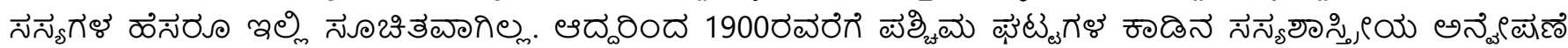

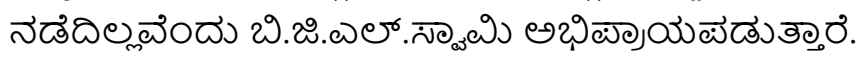

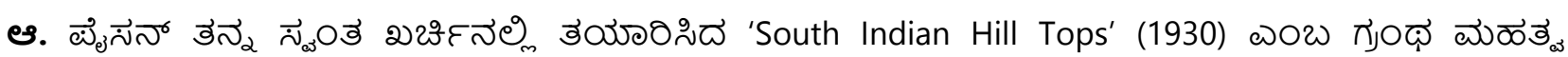

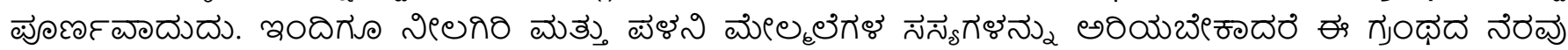

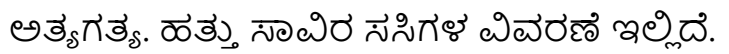

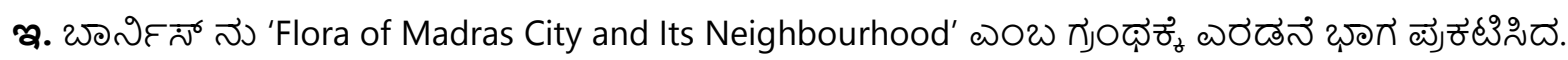

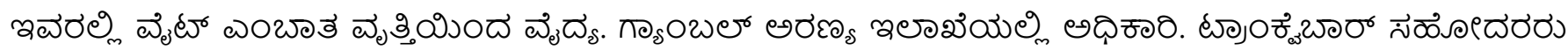

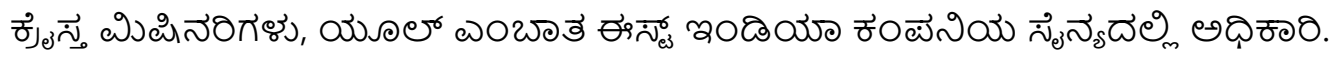

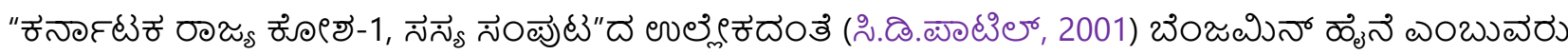

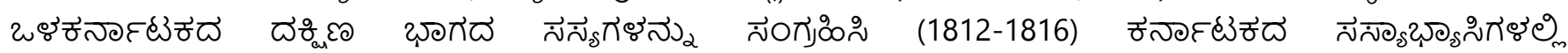

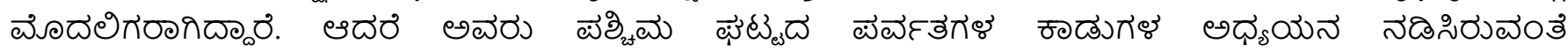

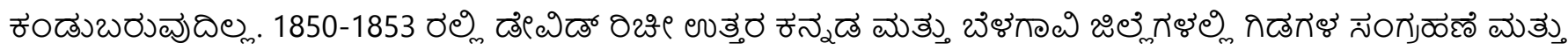

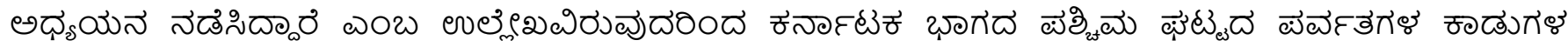

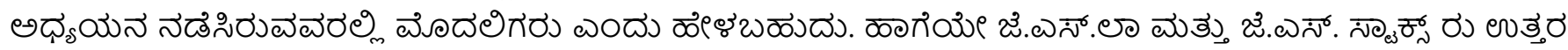

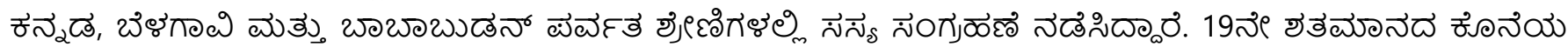

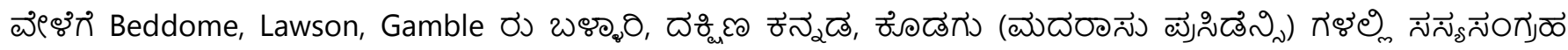

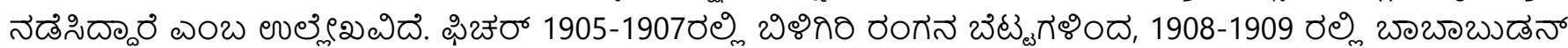

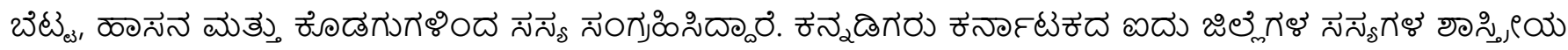

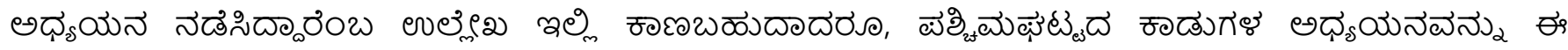

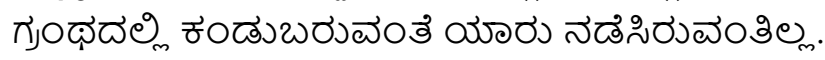

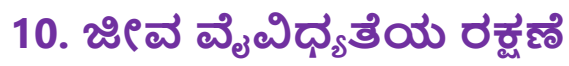

"జిథత్

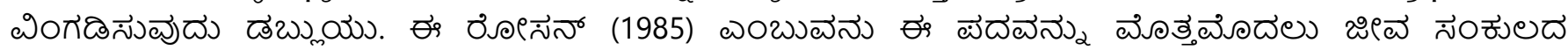

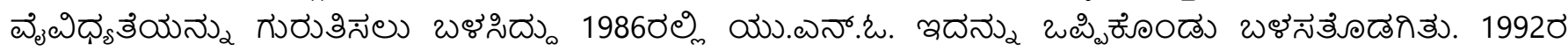

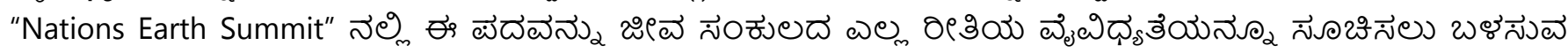
నిధాణర యోడెలాయితోు.

"The IUCN Red list of Threatened Species created on 1963, is the World's most comprehensive inventory of global conservation of nature and national resources. (IUCN) is the world's main authority on the conservation states of species. The IUCN Red list is set upon precise criteria to evaluate the extinction risk of thousands of species and subspecies.

The 2006 Red list released on $4^{\text {th }}$ May 2006 evaluated 40,168 species asa wholes plus an additional 2,160 sub species, varieties, aqutic stocks and subpopulations, From the species evaluated as a whole, 16,118 were considered threatened, of these 7,725 were animals, 8390 were plants and three were lichen and mushrooms (Rajeev, 2009)

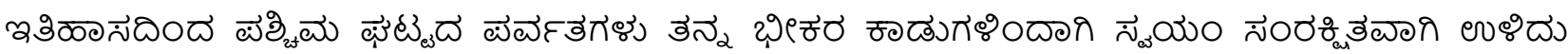

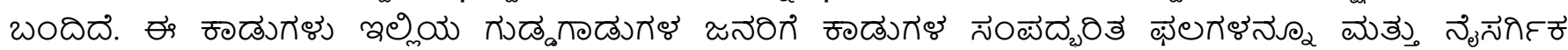

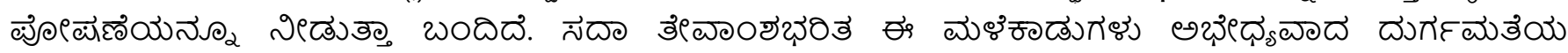

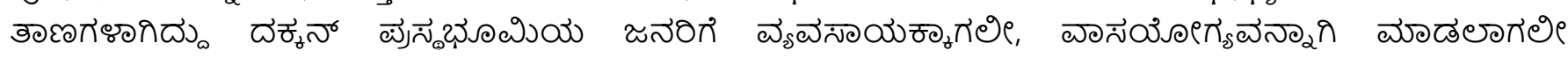

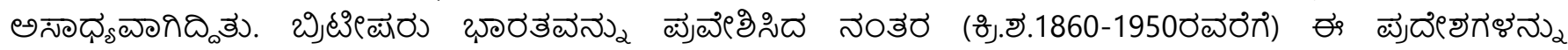

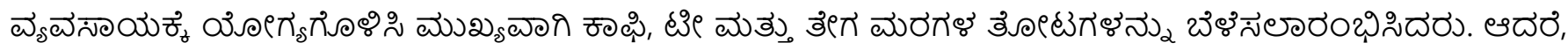
ఇంతळ

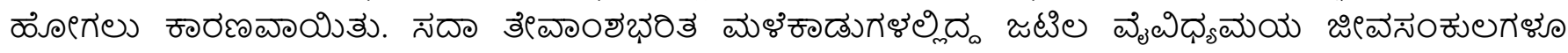




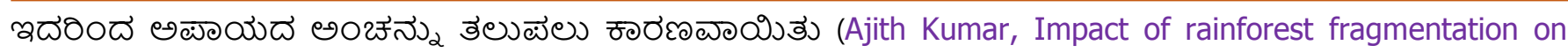
small mammals and herpetofauna in the Western Ghats, South India)

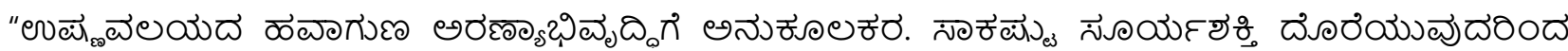

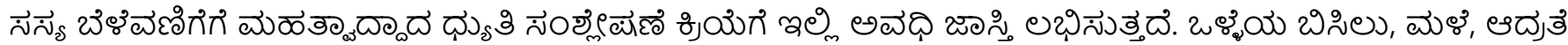

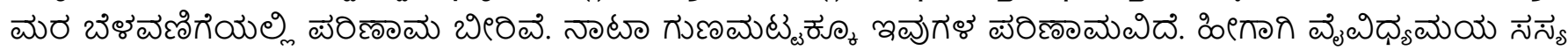

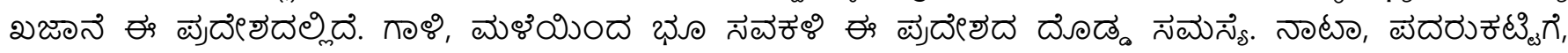

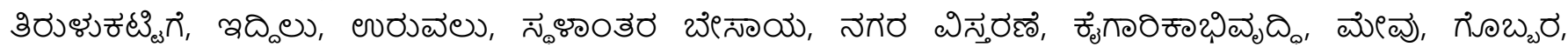

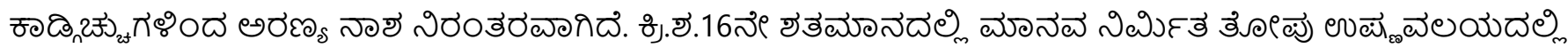

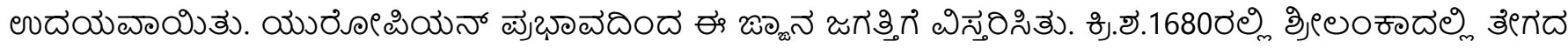

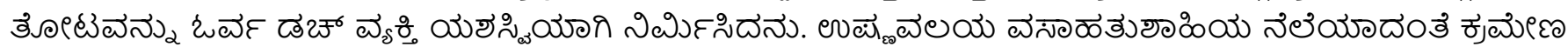

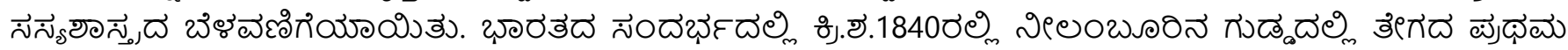

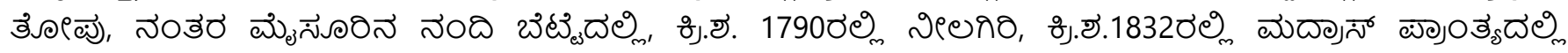

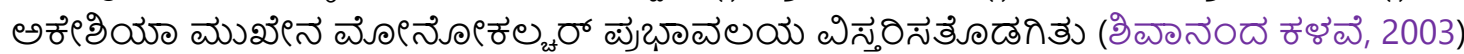

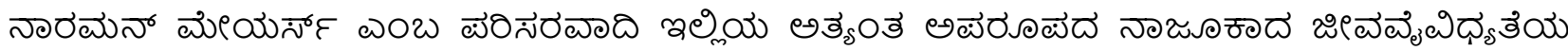

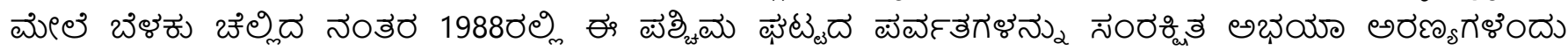

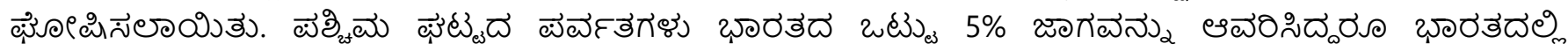

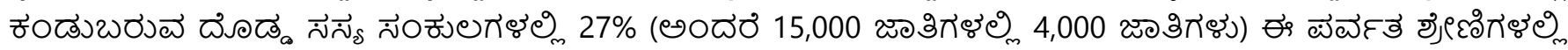

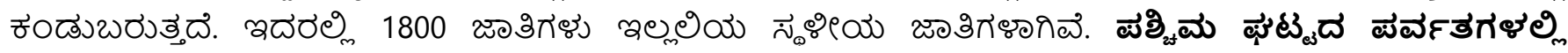

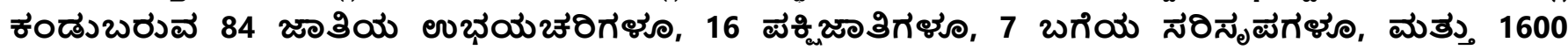

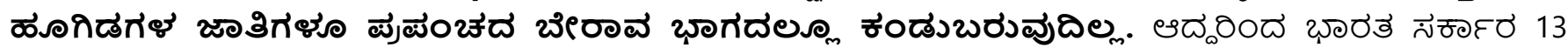
రాషిట్ట

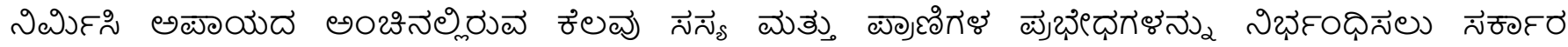

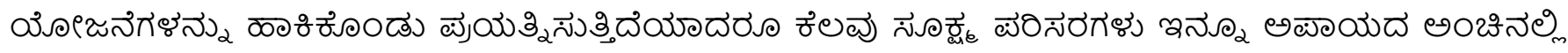
లుళిదృశீอండిఱె.

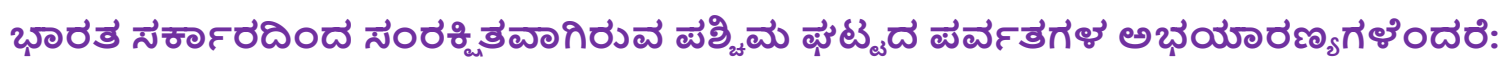

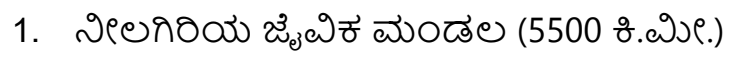

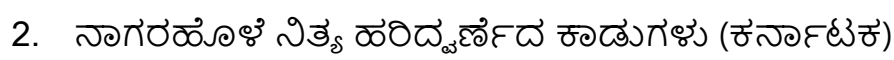

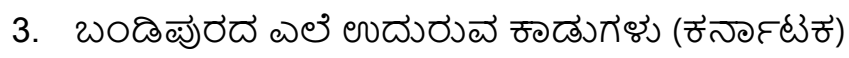

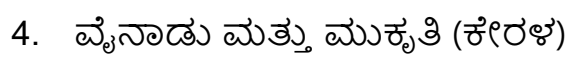

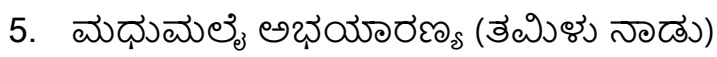

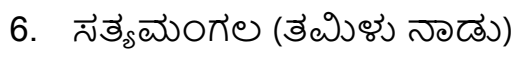

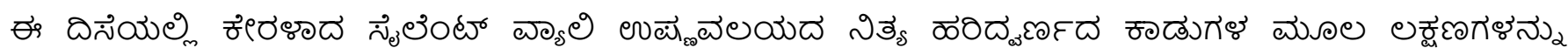

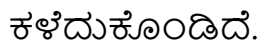

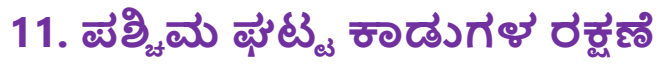

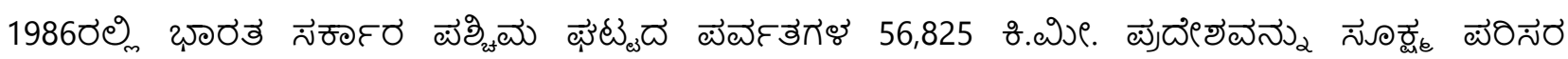

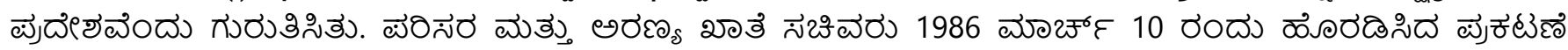

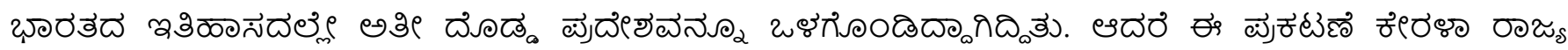

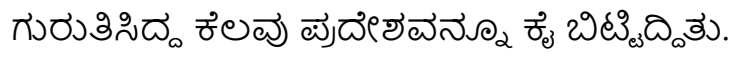

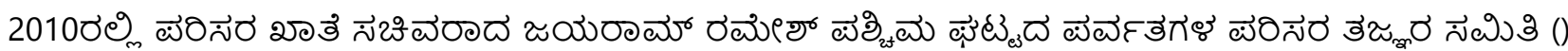
యను్ను

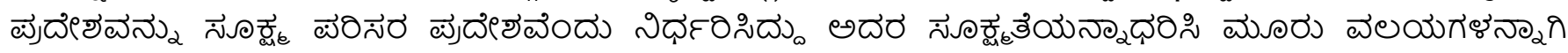

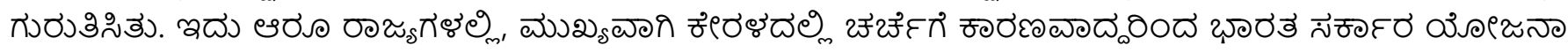




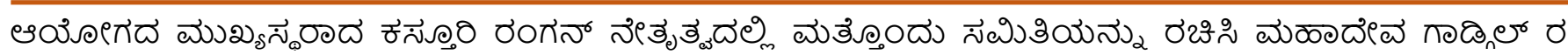
సెటికిియ థరరదియనున్న

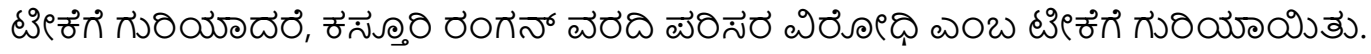

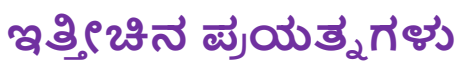

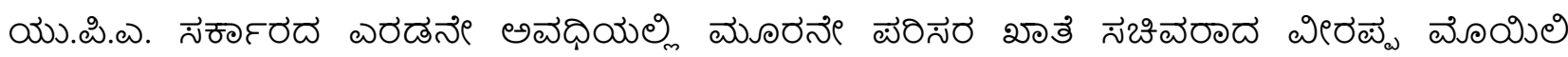

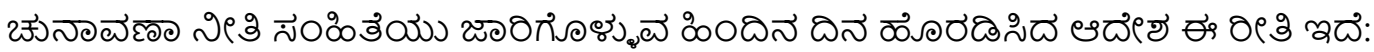

Government of India, Ministry of Environment and Forests Notification New Delhi, 10 ${ }^{\text {th }}$ March, 2014 S.O. $733(\mathrm{E})$ - The following draft of the notification:

"And whereas, taking into account the ESA demarcated by Kerala Government for the state of Kerala instead of Ecologocally Sensitive Area recommended by high level working group for the state, the total ecologically sensitive Aria for Western Ghars region works out.

Therefore, in exercise of the powers conferred be section 3 of the Environment (Protection Act, 1986 (29 of 1986) and sub-rule 5 of the Environment (protection) Rules, 1986, The Central Government here by notified the identified area of 56,825 sq km. Which is spread across six states namely Gujarath, Maharastra, Goa, Karnatraka, Kerala and Tamil Nadu as the Western Ghars Ecologically Sensitive Area (here is afer referred to as the Eco-Sensitive Area)"

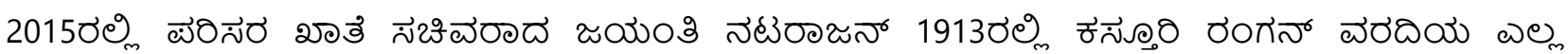

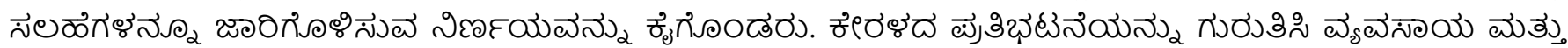

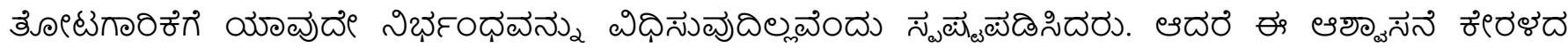

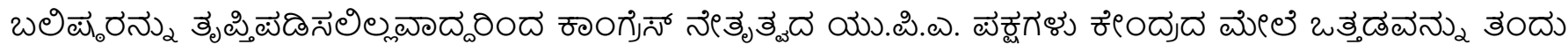

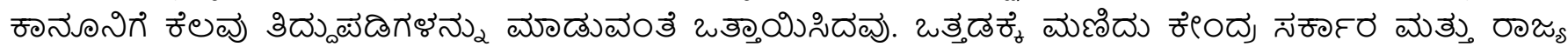

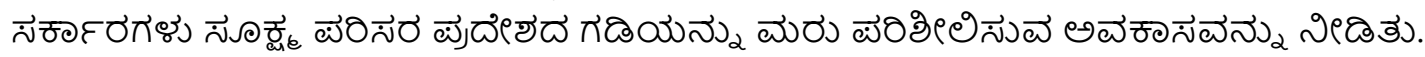

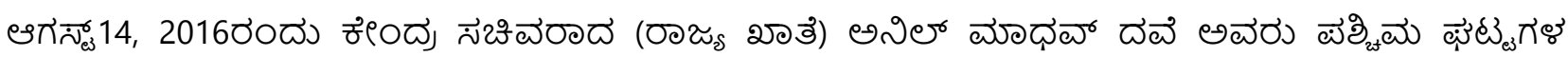

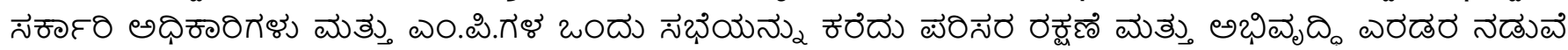

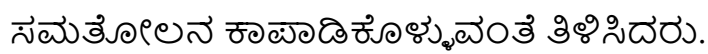

'ద దిందేల'

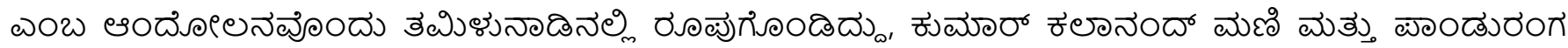

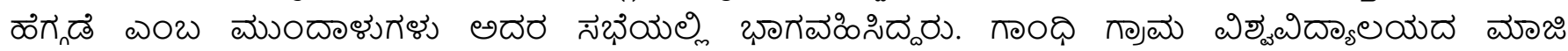

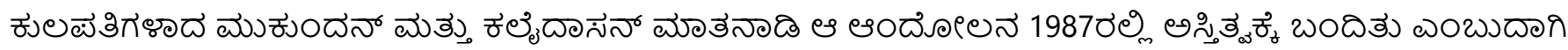

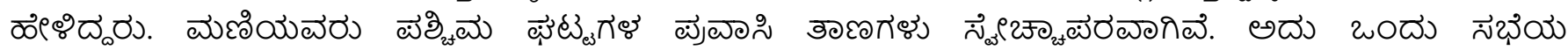

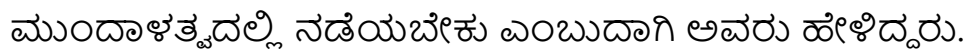

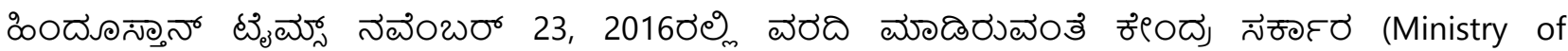
Environment, forest and climate change) "Mountain Policy" యను

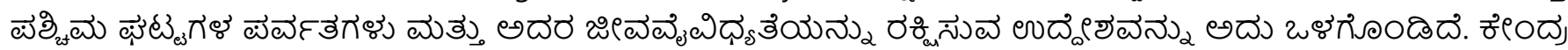

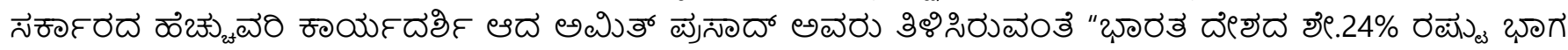

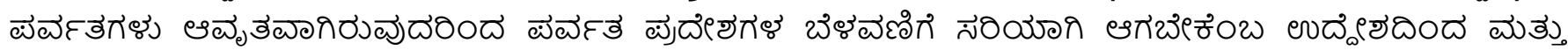

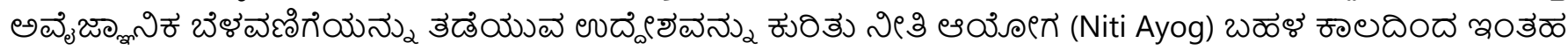

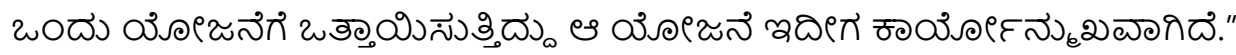

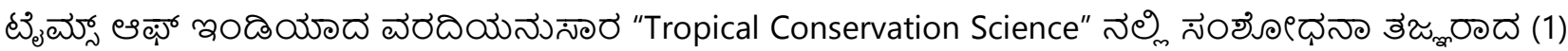

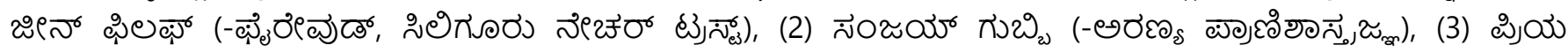

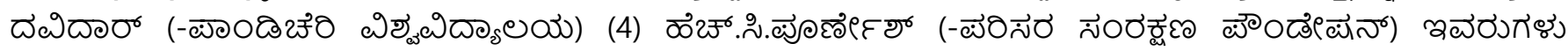

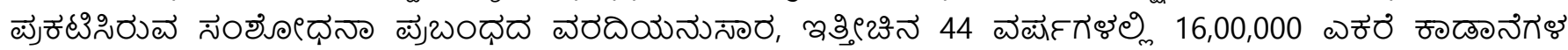
అరణ్య

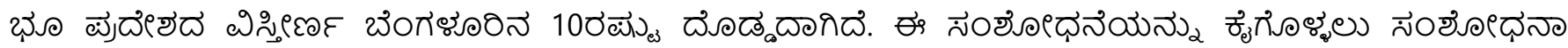

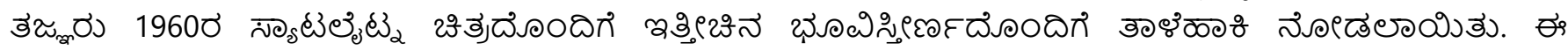

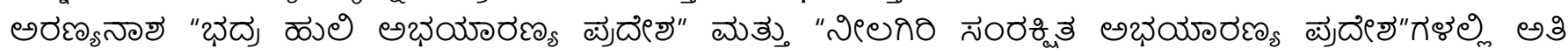




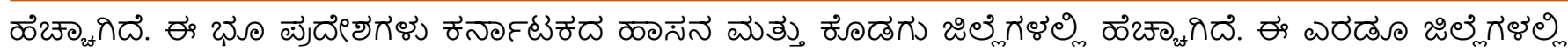

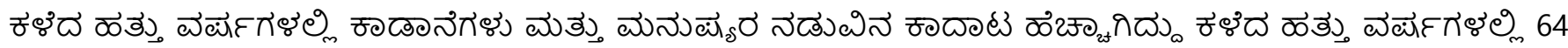

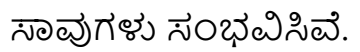

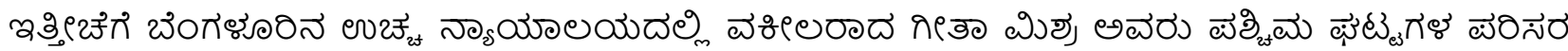

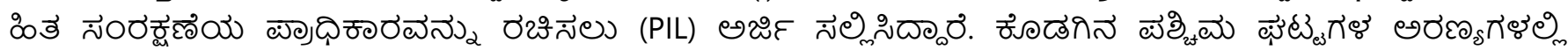

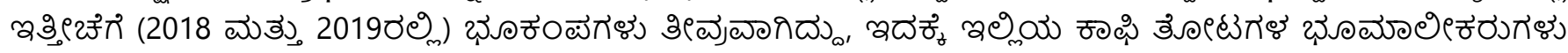

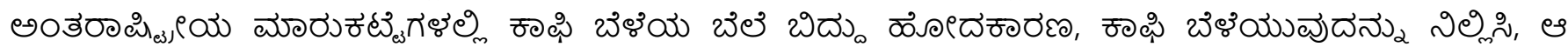

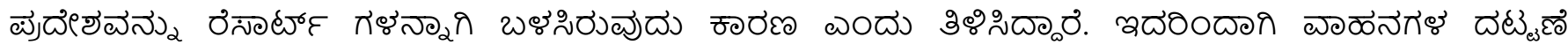

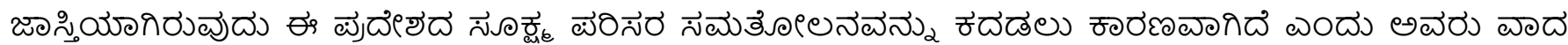

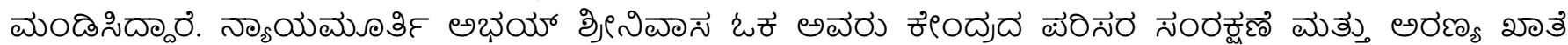

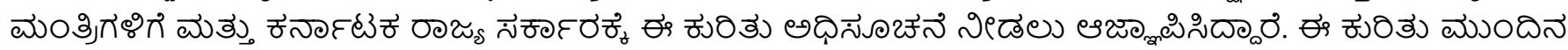

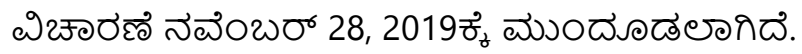

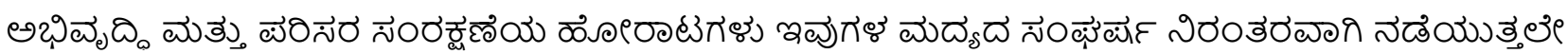

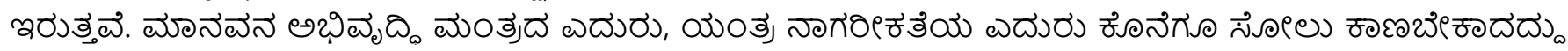

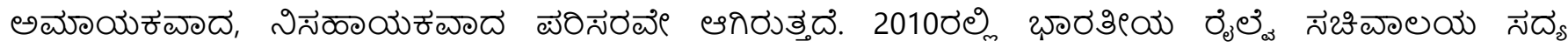

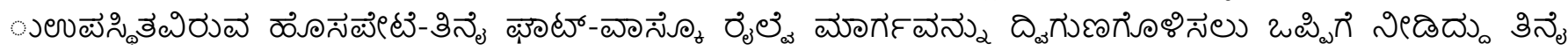

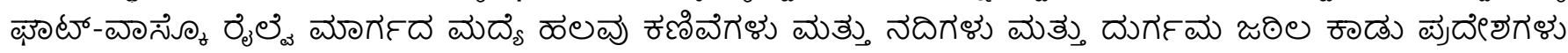

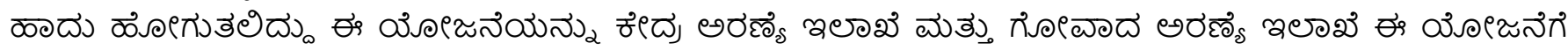

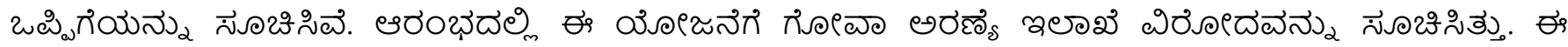

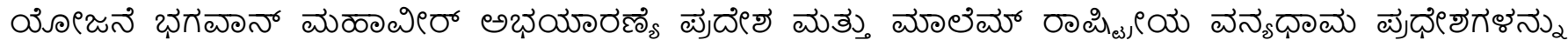

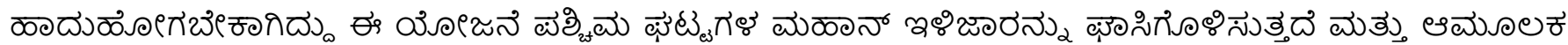

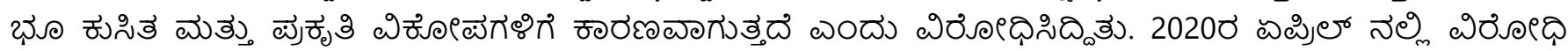

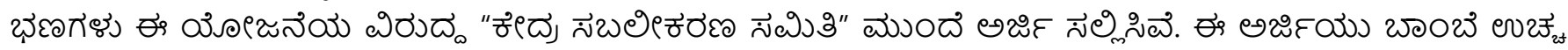

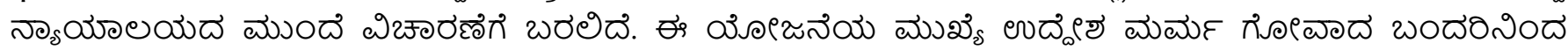

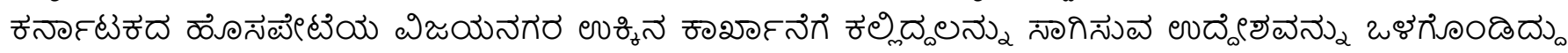

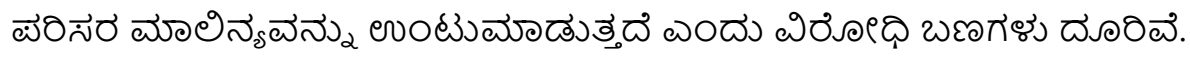

\section{References}

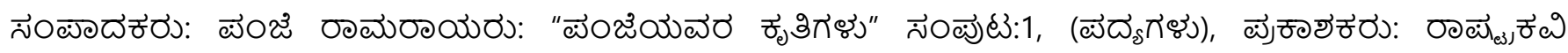

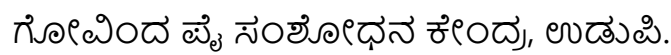

"Un designates Western Ghats as world heritage site". Times of India, 2 July 2012. Retrived 2 July 2012.

"The Peninsula": Asia-Pacific Mountain Network. Archived from the original on 12 August 2007.

Vijayan V.S. "Research needs for the Western Ghats". Ashoka Trust for Research in Ecology and the Environment (ATREE). Retrieved 21, June 2007.

Myers, N., Mittermeier, R.A., Mittermeier, C.G., da Fonseca, G.A.B., Kent, J., Boidiversity hotspots for conservation priorities, Nature, Nature, 403 (2000) 853-858. https://doi.org/10.1038/35002501

"Un designates Western Ghats as world heritage site", Times of India 2 July 2012, Retrived 2 July 2012.

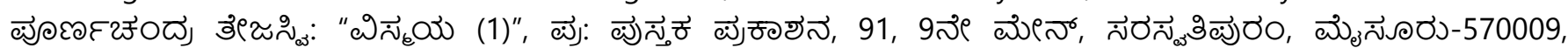

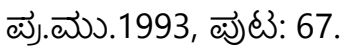

Ranjit Daniels, R.J., Biodiversity of the Western Ghats - An Overview, Wildlife. Institute of India.

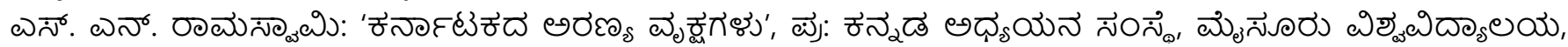

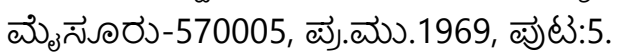

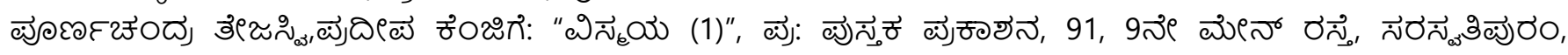

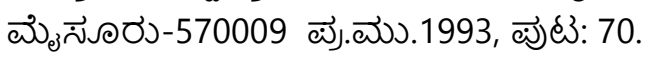

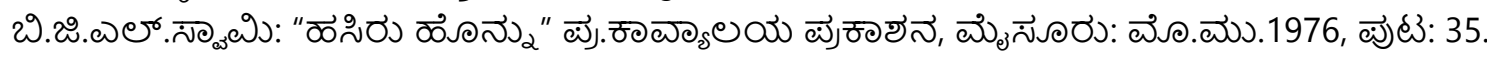

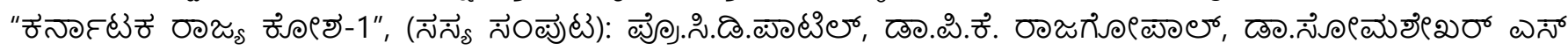

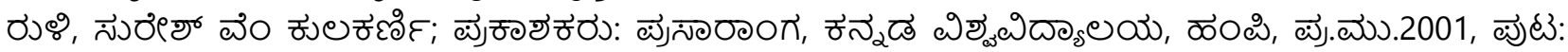

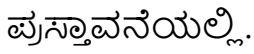

Rajeev, B.M.T., (2009) Glimpses of Indian Wildlife, (1 ${ }^{\text {st }}$ ed) Nekhara Prakashnana, Bangalore, India. Page No.3. 
Ajith Kumar, Impact of rainforest fragmentation on small mammals and herpetofauna in the Western Ghats, South India, Sakim Centre for Ornithology and Natural History, Coimbatore, Archieved from the original on 19 December 2008.

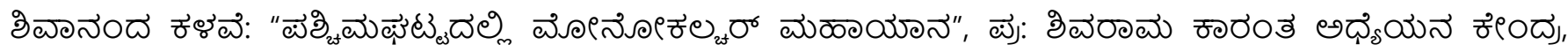

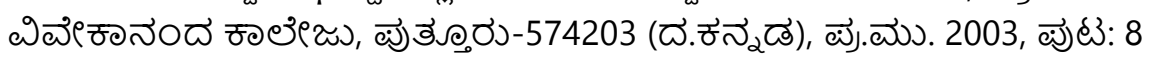

\section{Funding}

No funding was received for conducting this study.

\section{Conflict of interest}

The Author has no conflicts of interest to declare that they are relevant to the content of this article.

\section{About the License}

(C) The author 2021. The text of this article is open access and licensed under a Creative Commons Attribution 4.0 International License

\section{Cite this Article}

T.M. Geethanjali, A Scientific study of Forests of the Western Ghats, Indian Journal of Multilingual Research and Development, Vol 2, Iss 3 (2021) 52-64. DOI: https://doi.org/10.34256/ijmrd2137 